DIVISION OF THE HUMANITIES AND SOCIAL SCIENCES

CALIFORNIA INSTITUTE OF TECHNOLOGY

PASADENA, CALIFORNIA 91125

Two Information Aggregation Mechanisms for Predicting the Opening Weekend Box Office Revenues of Films: Boxoffice Prophecy and Guess of Guesses

David Court

AFTRS

Benjamin Gillen

Caltech

Jordi McKenzie

Macquarie University

Charles R. Plott

Caltech

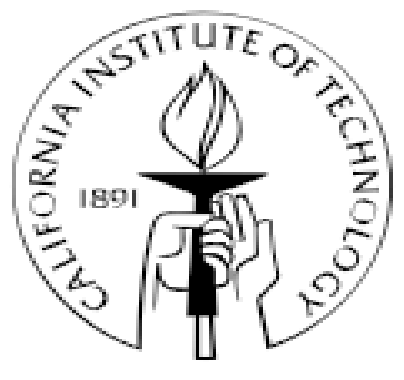

SOCIAL SCIENCE WORKING PAPER 1412

(December 28, 2015) 


\title{
Abstract
}

Two Information Aggregation Mechanisms for Predicting the Opening Weekend Box Office Revenues of Films: Boxoffice Prophecy and Guess of Guesses

\author{
David Court (AFTRS), Benjamin Gillen (Caltech), Jordi McKenzie (Macquarie University), \\ Charles R. Plott (Caltech) \\ January 2016
}

Successful field tests were conducted on two new Information Aggregation Mechanisms (IAMs). The mechanisms collected information held as intuitions about opening weekend box office revenues for movies in Australia. Participants were film school students. One mechanism is similar to parimutuel betting that produces a probability distribution over box office amounts. Except for "art house films", the predicted distribution is indistinguishable from the actual revenues. The second mechanism is based on guesses of the guesses of others and applied when incentives for accuracy could not be used. It tested well against data and contains information not encompassed by the first mechanism. 


\section{Two Information Aggregation Mechanisms for Predicting the Opening Weekend Box Office Revenues of Films: Boxoffice Prophecy and Guess of Guesses}

David Court (AFTRS), Benjamin Gillen (Caltech), Jordi McKenzie (Macquarie University), Charles R. Plott (Caltech)

December 2015

\section{Introduction ${ }^{1}$}

This paper reports a successful field test of two Information Aggregation Mechanisms (IAMs) that depart from traditional theory and institutions. The challenge we confront in this field test is to collect information about opening weekend box office revenues for movies in Australia. Our primary focus is on the performance of the IAMs when operating in an environment that departs from the controlled environments in which experimentalists refine and test the mechanisms based on known and fixed parameters. The question is whether the messages delivered by laboratory experimental methods regarding mechanism performance are robust to parameters as they are found to naturally occur in the world and if not, identify causes for the lack of robustness. This concern is among the classical questions regarding the relevance of laboratory methods for addressing problems found occurring naturally.

The movie box office test poses some challenges beyond those previously studied in field tests of IAMs. The field test requires a model of how information is distributed among people as well as how that information is reflected in decisions. The tests rest on hypotheses regarding both the underlying information available to IAM participants as well as how the collected information will be presented in IAM outcomes. The predictions derived from joint hypotheses about these environmental and institutional features are then tested against the actual distribution of observed box office revenues among the films. The results demonstrate that the mechanism performs substantially as predicted by the theory as it has been tested in highly controlled laboratory environments. The information about box office revenues exists to be collected. The IAMs collect it.

\footnotetext{
${ }^{1}$ We thank the Gordon and Betty Moore Foundation; the Lee Center; Australian Research Council (Linkage Grant LP110200336); University of Sydney; Australian Film, Television and Radio School (AFTRS); and the Caltech Laboratory for Experimental Economics and Political Science. The computer and software development skills of Hsing Yang Lee and Travis Maron are acknowledged. Their skills and dedication made the research possible. The comments of Matt Shum were very helpful.
} 
Information Aggregation Mechanisms (IAMs) are designed to collect and aggregate infomation held in the form of subjective beliefs and intuition dispersed among the participants. Their purpose is to quantize, collect, and organize information about the likelihood of specific events. This paper reports the results of a field application of IAM's as opposed to contolled experiments that test the ability of a mechanim to perform the task under laboratory conditions in which the information to be aggregated is known to the experimenter. Field tests explore the robustness of the IAM's ability to collect and aggregate information in environments that do not control all features of the mechanism itself, the participants' skills, the event studied, the information available about it or how it is distributed. In the field tests, neither the existence of the information nor its quality are known. The questions we must address are both (a) whether or not information exists to be aggregated and (b) whether or not the IAM is successful in gathering it.

Traditional IAM architectures are based on markets with Arrow-Debreu assets that can be traded in applications potentially lasting days or even weeks. Neither of the two architectures studied here follows that tradition. No trading takes place as participants' actions are irreversible, indeed markets are not even part of the mechanisms. The excercise occurs once and lasts about an hour or less. The architecture of the primary IAM is similar to parimutuel betting processes but explicitly designed to avoid the information distortions known to contaminate information aggregation associated with betting processes typically used for entertainment purposes. The second mechanism is completely different and is similar to the classical "guessing game" and explores the capacity to analyze events, such as those far in the future, or poorly defined events that cannot be used as a basis for structuring rewards for accurate information.

Not only are the architectures of the IAMs used here different from those typically used, the conditions of the applications are relaxed considerably. Traditional information aggregation mechanisms are deployed in environments in which the information flow is quantitatively based and available to IAM participants who understand the information, expect to detect it in the behavior of markets, and can act on it in the context of the IAM. Often IAM participants are associated with a business interest and have access to private, quantitative information related 
to the event of interest. By contrast, the participants in the application reported here were students associated with a film school as well as some industry professionals. Little or no quantitative information existed or was available to participants about the opening weekend box office of the movies. The information existed primarily in the form of subjective impressions and opinions based on the properties of the films and possibly the success of films that share similarities.

\section{A. The Nature of the Mechanisms}

Two mechanisms are studied. One is a parimutel based IAM and the second is similar to the "guessing game" (Nagel 1995).

In the parimutel based IAM, called Boxoffice Prophecy (BOP), the range of values that possible box office ticket sale quantities can take is partitioned into a set of non-overlapping intervals, or "buckets." The participants in an exercise are given an opportunity to purchase "tickets" and a cash prize is awarded to the holders of tickets when the variable of interest takes a value within a given bucket. Participants are allowed to buy as many tickets as they wish (up to a budget limit described below) and place them freely in any of the buckets. In this way, the distribution of tickets placed across the different buckets yields a potential measure of participants' beliefs regarding the future realization of the variable of interest. The information aggregation mechanism automatically aggregates these beliefs across participants, allowing the construction of "consensus" forecasts while also obtaining a glimpse into the underlying uncertainty.

The second mechanism directs participants to make a guess about the box office of an upcoming film. Each participant submits a single number anonymously as a guess. The actual box office will not be known within a time frame required to base payoffs on the box office when it becomes known. Indeed exactly how the boxoffice will be measured may not be known at the time of the IAM exercise since measurment methodologies evolve. Instead, a prize is awarded to the individual(s) whose guess is closest to the median guess of all other individuals. The mechanism will be called the Guess of the Guesses (GOG).

Clearly this mechanism is exploratory in that no generally accepted theory suggests success. Typical game theory applications focused on asymmetric information rest on 
assumptions about an individual's understanding of the rationality of others. An alternative perspective follows from the intuition that when uninformed about the opinion of others, individuals tend to use themselves as a model. Thus, when guessing what others will guess subjects are drawing on their own guess about the box office and rewarded if they are correct.

\section{B. Orientation and Outline}

Section 2 will focus on details of the two mechanisms, their structure, and how participants interact with the mechanisms. Basically, the section answers questions related to what participants see and what they do. Section 3 contains background material. Section 4 outlines the procedures, subjects and timing of the study. Section 5 is a discussion of the movies for which the box office is to be predicted. Section 6 introduces a model of the information environment. The section develops a general model of the information, how it is expressed and what it means to aggregate it. In this model the predicted event is not a "state" but a probability distribution and the results of the IAM are interpreted as the probability of various box office amounts. Section 7 contains results. The performance of the two IAMs are evaluated and compared. Section 8 is a summary of conclusions.

\section{Two Information Aggregation Mechanisms: Institutional Frameworks}

The purpose of an Information Aggregation Mechanism (IAM) is to quantize, collect and aggregate information held in the form of subjective intuitions held by different individuals about uncertain future events. Presumably, these events are accompanied by different forms of information distributed across a population that can be represented as differentially and independently distributed signals.

The two mechanisms studied, 'Boxoffice Prophecy' and 'Guess of the Guesses', differ in structure and background theory. Information aggregation models (IAMs) such as Boxoffice Prophecy (BOP), rest on a hypothesis that the collection and aggregation of such information produces a combined signal that has more information content than any single signal. The mechanism produces a probability distribution that becomes interpreted as the aggregation of the existing information. The Guess of the Guesses (GOG) model is not so much an attempt to aggregate information as it is an attempt to extract an individual's information under conditions 
that prohibit the use of incentives based on accuracy of reports or guesses. The output can be interpreted as the distribution of modes of opinions.

\section{A. The Boxoffice Prophecy (BOP) Mechanism}

Participants interact with the mechanism in the form of an on-line, interactive program. They can log in at the scheduled time and participate from any computer with an appropriate internet connection. The process operates in real time during which they are able to place small "bets" on various levels of box office amounts. Figure 1 is a screen shot of the interface used by participants in the BOP exercises. The illustration is for a single film.

\section{Figure 1. Screen Shot of BOP Interface}

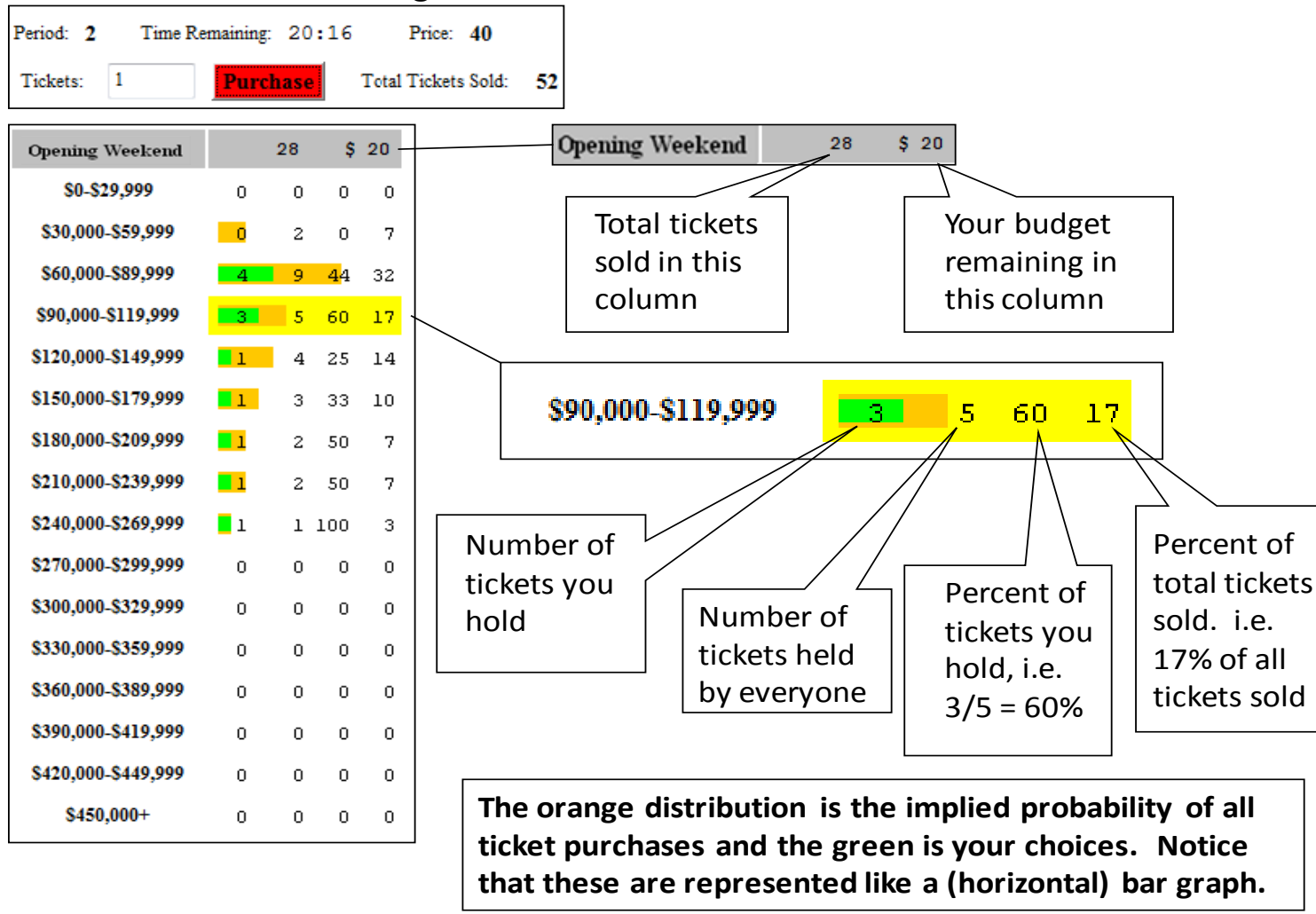

The participant places the curser on a bucket and clicks. The spaces turn yellow as is the case of the $\$ 90,000-\$ 119,999$ bucket in the figure. By clicking on the PURCHASE button the individual purchases a ticket in that bucket. The price shown in the figure is 40 while the budget for this participant is currently 20 so the system would refuse the purchase due to insufficient cash. Had the purchase been made the number of tickets held by the individual would go up by one as would the number of tickets held by everyone. Percentages would be adjusted accordingly. 
For a given film the potential opening weekend box office amount is partitioned into intervals that we will refer to as "buckets". ${ }^{2}$ For description purposes we will consider a single variable, say opening weekend box office for film $i$ in week $t$, that we denote by $Y_{i, t}$. The positive real line is partitioned into $K$ intervals, the "buckets," where each interval represents a range of possible values for the opening weekend box office that will be officially reported when the movie opens. The leftmost and rightmost buckets are, respectively, $\left[0, x_{1}\right)$ and $\left[x_{K-1}, \infty\right)$. The bucket sizes for the example illustrated in Figure 1 are \$29,999.

Mechanism organizers invite participants, who securely log in to their own account to access the IAM program. The mechanism makes "tickets" available for sale to participants, who spend an endowment of Francs (our synthetic experimental currency) on tickets and allocate them across the buckets. At the opening of each application all participants are given a fixed budget of 500 Francs for each of the predicted variables. The Francs cannot be transferred among participants, used in other applications, or assigned to buckets for another film's BOP excercise. Each BOP session operates at a fixed time and only those invited are able to participate. The IAM program stores a wealth of data, including individual participant actions and time-stamps indicating when each of these actions took place.

The tickets for all buckets are priced the same and that price will increase at a preannounced rate to ensure the mechanism closes in a reasonable time. An example of the price is displayed in Figure 1. For example, the opening price would be constant for fifteen minutes and then go up at a rate of one Franc per minute after that. These price changes discourage waiting until the last second to purchase, helping to offset individual incentives to hold back their private information and to improve their own information by learning from others' decisions. All participants are aware that their own information might be improved through seeing the purchases of others. They are also aware that their own information might be

\footnotetext{
${ }^{2}$ The number of buckets is dictated by screen size. The size of the buckets is determined by how the film is classified. "Art House" films have the smallest buckets, followed by "Regular" and then "Blockbuster" which is the largest. The movies, classifications, and buckets are discusssed in Section 5.
} 
communicated by their own purchase of tickets. The temporal discounting helps to mitigate these strategic incentives that otherwise hinder successful information aggregation.

Throughout the operation of the mechanism, participants have a continuously available record of the number of tickets that are currently placed in each of the buckets. At each instant during the application, as well as at its termination, the placements of all tickets in all buckets are known as is illustrated in Figure 1. The individual participant also knows the proportion of tickets he or she holds in each bucket, which is particularly important because these proportions are the foundations for incentives. When the actual winning bucket becomes known those holding tickets in that bucket are given a part of a grand prize equal to the proportion of the winning bucket tickets that he or she holds. If participant $n$ holds $z \%$ of the tickets sold for the winning bucket then participant $n$ gets $z \%$ of the incentive prize. For example, if the incentive prize was $\$ 1,000$ and the individual held $10 \%$ of the tickets sold for that bucket then the payment to participant $n$ would be $\$ 100$.

A typical BOP exercise involved forecasting two or three films. The exercise takes place once a week and requires on the order of 30 minutes with the maximum possible being an hour. Each participant is given a separate Franc budget for each film they forecast. All budgets are the same size and the budgets are not fungible across the items forecast. The number of participants typically ranges from ten to twenty and each operates from a secure computer located wherever the participant happened to be located (home, office, traveling, etc). Typically the users are anonymous within the mechanism: both the list of participants and the winners remain secret. Of course, the total of tickets purchased in each bucket of each forecast is public and known in real time as the tickets are purchased.

\section{B. Guess of Guesses (GOG) Mechanism}

In sessions focused on the Guess of the Guesses mechanism, participants log in to the BOP session and before proceeding further are asked to make a guess about the opening box office of films that will open several months in the future. The horizon is beyond a time interval within which the realized box office data could practically be available for payment, implying the opening box office will not be known so cannot be used. Nevertheless, information could 
be available in the same form that becomes available for films in general. Of course, the guess cannot be made incentive compatible in the traditional sense of the term as realized accuracy of a guess cannot be the basis for a reward.

The GOG is based on the hypothesis that the best information about what others might believe is introspection. The intuition is that when uninformed about the opinion of others, inidividuals tend to use themselves as a model. ${ }^{3}$ Thus, when guessing what others will guess they are drawing on their own guess about the box office and rewarded if they are correct. That is, the best model a person has about the beliefs of others is his/her own beliefs. Thus, the GOG procedure rewards the individual according to the accuracy of their guess of others. Clearly, this has a potential for unconstrained possibilities as the infinite regress of the average thinking what the average thinks. Nevertheless, we implement the mechanism and test its performance.

Clearly the GOG mechanism is exploratory in that a formal theory that predicts success of the model does not exist. Figure 2 is the instruction given to subjects. Each subject is asked to make a guess about the box office of the film. However, the reward is given to the guess that is closest to the median of the other guesses. In case of ties, the reward is split.

\footnotetext{
${ }^{3}$ This idea was motivated by insights contained in Prelec (2004) and Weaver and Prelec (2013). The property could be a consequence of an "availability heuristic" or, alternatively a "recognition heuristic". The idea also appears as a substantive principle in "false consensus" research. We use the property axiomatically take no stand on competing explanations or the conditions under which it might be reliable as a model.
} 


\section{Figure 2 - Screen shot for GOG Mechanism}

The first pages of Boxoffice Prophecy ask you for your opinion about others' opinions. You are not paid on the accuracy of your answer with respect to the actual boxoffice. You will be paid if you are the closest to the average (actually median) answer given by others.

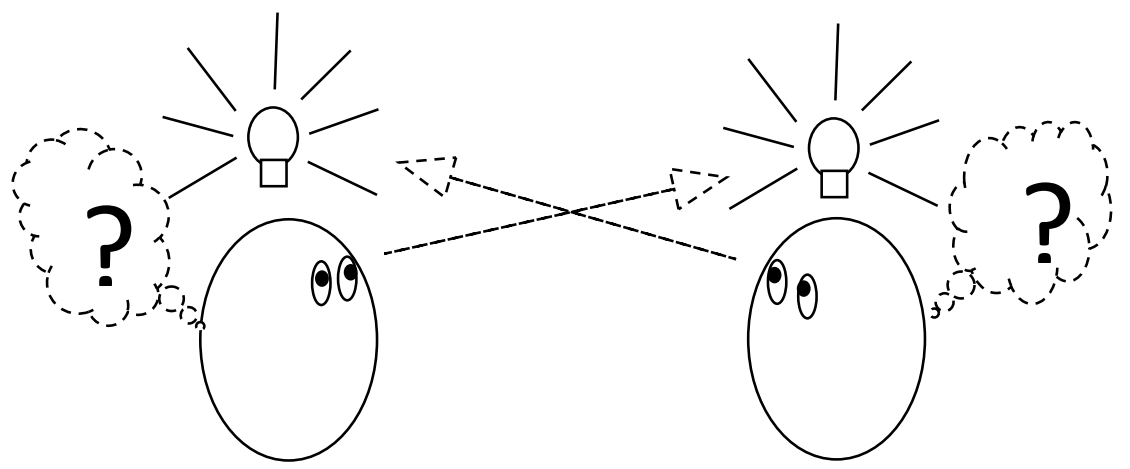

Notice that we are NOT asking you what you think the boxoffice will be. We are asking the individuals in the group what they think that the average (median) of the group thinks. What do you think that the average (median) person of the group thinks that others think? Your personal thoughts about the boxoffice could be very different. Think for a minute.

\section{Background}

For decades, economic theory has explored the theory that markets collect and aggregate information contained in prices. Motivated by the rational expectations and finance literature Plott and Sunder $(1982,1988)$ produced the first experimental demonstration that market equilibration and associated prices can perform an information aggregation function. The idea that markets could be designed and implemented to aggregate important information followed almost immediately with an application to sales forecasting (Chen and Plott, 2002; Plott, 2000) and election predictions (Berg, et al. 2008). These market based applications were followed by of numbers of papers and interest sufficient to attract the attention of the Commodities Futures Trading Commission.

While the feasibility of IAMs and their application to important problems is established, practical challenges are abundant. Market-based IAMs and prediction markets face practical challenges inside businesses. Real time, multiple markets of Arrow-Debreu securities require training on tendering the bids and asks; inventory and cash management; and information 
management of time series of order flow data. In addition, market participation requires time. Management of markets requires infrastructure and time for posting results, constant encouragement for participation and technology repairs. Basically, the operation of continuoustime multiple markets is costly.

In a series of laboratory experiments, Plott, Wit and Yang (2003) discovered that parimutuel betting systems are capable of performing information aggregation but the performance levels are poor. A long series of research papers isolated institutional improvements resulting in the architecture used here. ${ }^{4}$ Continuous time order flow was shown to be important for information aggregation in the first market experiments and was retained in the parimutuel based IAM. A tendency for participants to postpone ticket prices until final moments and free ride on information reveled by others was corrected by implementing an increasing price of tickets thereby making purchase delays costly (Axelrod, et al.2009). The speed of price increase became a tool to limit the time devoted to the exercise. The time required for participation and the timing of participation both reflected the opportunity cost of participation. The risk that is implicit in parimutuel betting, had a tendency to inhibit ticket purchases and limit the inflow of information into the mechanism. The participation risk was corrected by removing the self-financing feature of parimutuel betting was replaced with a prize to winning bucket ticket holders in proportion to holdings. Ticket purchases were in terms of a synthetic currency that had no value outside the experiment and initial endowments of currency given to participants. Thus, the participants had an incentive to spend their entire budget and risk aversion was minimized as a barrier to participation (Plott and Roust, 2009). Furthermore, relative purchasing power among participants and the selection of participants become controlled. Carefully constructed screens and instructions were needed to facilitate a quick and accurate understanding and avoid misconceptions about a mechanism that was

\footnotetext{
${ }^{4}$ Papers by Axelrod, et.al. (2009) and Plott and Roust (2009) added both understanding and features. Axelrod, et.al. (2009) demonstrated that the addition of a time clock and an increasing price of parimutuel tickets would increase the speed with which information flowed into the system. Plott and Roust (2009) demonstrated that poor information aggregation was related to weak signals. When the signals are weak, information aggregation is poor, primarily because risk aversion prevented agents acting on poor information. That information exists to be reflected in the decisions of others is not revealed. Those two studies lead to the major features of the architecture implemented in Intel and in Boxoffice Prophecy. More recently the results of Kalovcova, et al. (2009) and Koessler, et al. (2012) have added depth to the understanding.
} 
otherwise very foreign to participants. As a result of the sequence of changes the IAM efficiencies improved dramatically.

The first field application of the parimutuel IAM was conducted inside Intel Corporation (Gillen, Plott and Shum, 2015). Among the many potential Intel applications those chosen existed in environments considered as good candidates for successes. Indeed, the performance of the IAM inside Intel is impressive in terms of assessing the probability of events and performance levels exceeding those of Intel official forecasts. The events were quarterly sales of well-defined categories of products. Past statistics were available as were quantitative measures, formal models and continuous updates of flows of indicator variables thought relevant. Participants were experienced sales personnel and managers with detailed knowledge of the business and had substantial experience working with the mechanism and understanding its accuracy.

By contrast, as a robustness test the box office application goes beyond the Intel application in terms of (i) the inexperience of the participants (Intel participants had years of experience and feedback with the mechanism focused on the same or similar commodities while the subjects in the BOP had at most 20 decision sessions in which 77 different film box offices were considered); (ii) the poor quality of the information available to participants (Intel participants had flows of quantitative data, including statistical models, from a variety of sources that held information about upcoming sales while the BOP subjects had no systematic flow of data about the Australian box office of any particular movie.)

The motion picture industry provides an example of products (i.e. movies) that are notarized for high levels of uncertainty (De Vany, 2004). The ability to predict box office revenues has proved challenging but studies reveal the subtle sources where it might exist. Useful surveys of econometric and related studies are provided by Eliashberg, et al. (2006), Hadida (2008), and McKenzie (2012). Other researchers have utilized data from online discussion forums and social networks to examine box office predictability. Mishne and Glance (2006) examine sentiment from weblogs. Doshi, et al. (2009) use movie ratings (IMDb users and Rotten Tomatoes critics), social network analysis on websites and blogs, and sentiment analysis of IMDb forum posts. Asur and Huberman (2010) develop a model based on Twitter 
posts (tweet rate per day over the week prior to release). Metsyan, et al. (2013) study data from Wikipedia editors and users. Recently, Google (2013) have also released a white paper documenting a predictive relationship between search volume and opening weekend box office revenues.

An ongoing market-based prediction exists as the Hollywood Stock Exchange (HSX), a virtual stock market where participants trade stocks tied to box office revenue outcomes. A number of academics have noted that the prices correlate with actual box office outcomes including Pennock, et al. (2001), Spann and Skiera (2009), Foutz and Jank (2010), and McKenzie (2013). Another (discontinued) industry based process developed by the popular Box Office Mojo movie website existed as the Box Office Derby and involved participants guessing box office for selected movies each week. McKenzie (2013) found the aggregated guesses are frequently close to the actual box office as well as being very highly correlated with the HSX predictions.

It is tempting to conclude that the information about box offices exists and is high quality but several features suggest that existing tests might not be particularly challenging. Predictions from HSX and Box Office Derby tend to be within the final week or, in the case of the HSX, the final closing price the day prior to release. ${ }^{5}$ These mechanisms only consider movies with wide screen distribution, whereas smaller movies like art houses are not in the mix. Also, the HSX is widely known to have thousands of registered participants and McKenzie (2013) notes the Derby had 350-800 participants per week over his sample period. Anecdotally, it is also known that many of the HSX and Derby participants were employed within the industry and possibly operated under incentives that differed from simply predicting the box office. Everything considered, the studies certainly suggest the existence of information but its quality and the power of these mechanisms to collect it are questions that require study.

\section{Procedures}

Boxoffice Prophecy (BOP) and Guess of Guesses (GOG) sessions took place in a series of stages within the calendar years 2006, 2008, 2010, and 2012 as was dictated by the available of

\footnotetext{
${ }^{5}$ Foutz and Jank (2010) is an exception who investigate the price path to assist in forecasts of box office revenues.
} 
funding. A total of 77 films were studied in 37 sessions resulting in 118 forecasts. Over this period 167 participants were involved and paid a total of approximately $\$ 62,000$ (AUD). The summary statistics are contained in Table 1.

Table 1. Number of Sessions, Films, Participants and Payments

\begin{tabular}{lllllll}
\hline & Weeks of & No. & No. BOP & No. GOG & No. & $\begin{array}{l}\text { Approx. } \\
\text { Period }\end{array}$ \\
Operation & Films & Forecasts & Forecasts & Participants & Payments \\
\hline 2006 & 16 & 16 & 32 & 0 & 75 & $\$ 20,000$ \\
2008 & 3 & 3 & 6 & 0 & 13 & $\$ 1,500$ \\
2010 & 8 & 17 & 24 & 24 & 37 & $\$ 19,500$ \\
2012 & 11 & 41 & 22 & 20 & 42 & $\$ 21,000$ \\
Total & 37 & 77 & 84 & 41 & 167 & $\$ 62,000$ \\
\hline
\end{tabular}

Subjects were a mix of current and former students of the Australian Film, Television and Radio School (AFTRS) as well as a small number of industry professionals working in various capacities within the industry (e.g. theatrical distribution or exhibition). Some of the students were actively engaged in film production and other parts of the industry while others were full time students. Potential subjects were contacted through lists of existing and previous students and instructed to log into the Boxoffice Prophecy website and register in the database if they were interested in participation. Once in the database all communication was by email and regular updates to the website.

The website had instructions for participation (Appendix B), a practice website that allowed practice alone or with others, a schedule of films together with standard release information about the film, and a list of frequently ask questions. The practice website had examples of historical applications including ticket purchase for different box office amounts for different movies together with the final box office. Thus, the participants had evidence that the ticket sales can carry information. The instructions included perspective about mistakes typically associated with confusion with questionnaires to which they are often exposed and confusion with gambling systems. For example, they were told that the task they were asked to perform was not to tell us their opinion about the film, its artistic quality or its social value. The task was to make as much money as possible given their information and the decisions of 
others. They were also told that dominated strategies could be viewed as mistakes. In the BOP exercise, possible examples included putting all funds in one bucket when the subject did not think that bucket would win with certainty; putting nothing in buckets that were likely and for which ticket sales were low; buying multiple tickets in a bucket in which no one else is buying. The dominated of the latter follows from the fact that if only one ticket is sold in a bucket the prize goes to the holder of that ticket so multiple tickets have no extra value if a buyer is alone. Over the weeks of each iteration, the results of previous BOP exercises including the investments in buckets and final box office outcome were added to the website.

Subjects were given private links to their own earnings. Payment was by deposit to their bank account. The website also had weekly information links that summarized the results of the previous week. The potential prizes and payoffs were announced in the event that films were cancelled or other unexpected events occurred. They were told and reminded that a significant amount of prize money was available each week.

\section{Movies, Characteristics and Measurements}

The 77 films we analyze are listed in the table of Appendix A, which categorizes films according to (i) the Boxoffice Prophecy (BOP) and/or Guess of Guesses (GOG) date; (ii) the release date of the film; (iii) the bucket size for the particular title (see Table 2 and discussion below); (iv) the film type used in the empirical analysis (Art House, Regular, or Blockbuster); (v) revenue definition (opening day, opening weekend or total theater run); (vi) list of BOP measurements; and (vii) list of GOG measurements. Art House films are typically released by small studios while blockbusters are released by major studios backed by large production budgets and publicity. These terms are well-known to experts in the industry and films are readily classified accordingly, with the range of possible box office revenue suggested by historical experience.

Table 2 lists the number of buckets and size used for the different movies. The number of buckets was always 16, dictated by interface screen size, judgments about instructions and other subjective issues associated with experimental design. The range of the buckets tended 
to be the same for similarly classified films. However, over the course of the trials some adjustments were made reflecting issues related to the film and economic conditions.

Table 2. Film Classification, Bucket Size, and Range

\begin{tabular}{llllllll}
\hline \multirow{2}{*}{ Iteration } & Bucket & Revenue & No. & Lower & Bucket & $\begin{array}{l}\text { Upper } \\
\text { Bucket Size }\end{array}$ & $\begin{array}{l}\text { Fo. } \\
\text { Dilms }\end{array}$ \\
\hline $2006 \&$ & Small & Weekend & 16 & {$[0, \$ 49,999]$} & $\$ 49,999$ & $\$ 750,000+$ & 11 \\
2008 & Small & Total & 16 & {$[0, \$ 499,999]$} & $\$ 499,999$ & $\$ 7,500,000+$ & 11 \\
& Large & Weekend & 16 & {$[0, \$ 199,999]$} & $\$ 199,999$ & $\$ 3,000,000+$ & 8 \\
& Large & Total & 16 & {$[0, \$ 1,999,999]$} & $\$ 1,999,999$ & $\$ 30,000,000+$ & 8 \\
\hline 2010 & Small & Weekend & 16 & {$[0, \$ 29,999]$} & $\$ 29,999$ & $\$ 450,000+$ & 6 \\
& Large & Weekend & 16 & {$[0, \$ 799,999]$} & $\$ 799,999$ & $\$ 12,000,000+$ & 11 \\
\hline 2012 & Small & Weekend & 16 & {$[0, \$ 19,999]$} & $\$ 9,999$ & $\$ 160,000+$ & 2 \\
& Small-Med & Weekend & 16 & {$[0, \$ 49,999]$} & $\$ 24,999$ & $\$ 400,000+$ & 8 \\
& Med-Large & Weekend & 16 & {$[0, \$ 499,999]$} & $\$ 249,999$ & $\$ 4,000,000+$ & 6 \\
& Large & Weekend & 16 & {$[0, \$ 1,499,999]$} & $\$ 749,999$ & $\$ 12,000,000+$ & 5 \\
& Extra-Large & Weekend & 16 & {$[0, \$ 2,999,999]$} & $\$ 999,999$ & $\$ 16,000,000+$ & 1 \\
\hline
\end{tabular}

\section{A Model for Information Aggregation in BOP and GOG}

In order to evaluate the performance of the Boxoffice Prophecy (BOP) and Guess of Guesses (GOG) mechanisms, we develop a formal model of the information available to participants, how that information influences participants' beliefs, and how it relates to realized box office revenues. This characterization represents the aggregated information held by participants as a sufficient statistic reporting the expected likelihood of different possible box office ticket sales levels. We then model how incentives can guide participants to an equilibrium outcome in the BOP and GOG mechanisms that accurately aggregates this information. While other equilibria for the mechanisms might exist, the theoretical results establish that the observed success of these mechanisms as reported in laboratory environments is not inconsistent with theory and thus suggest the interpretation and application to other field environments. 


\section{A. Individual Information and Beliefs when the State of Nature is a Distribution}

Boxoffice Prophecy and the Guess of Guesses mechanisms attempt to forecast an outcome that is subject to multiple tiers of uncertainty. Not only are participants uncertain about what final sales will be, they are uncertain about the conditional distribution from which realized sales will be drawn. This higher-order uncertainty requires describing a stochastic environment with aggregate uncertainty both in realized sales and in the conditional distribution over sales.

For ease of exposition, denote realized sales for movie $t$ by $Y_{t}$ and suppose the potential values of realized sales are restricted to a discrete set of $K$ different values, $X=\left\{x_{1}, \ldots, x_{K}\right\}$, so that the distribution of realized sales can be represented by a multinomial distribution. Specifically, letting $\pi_{k}=\operatorname{Pr}\left\{Y_{t} \in x_{k}\right\}$ and conditioning on $\pi=\left[\pi_{1}, \ldots, \pi_{K}\right]^{\prime}$, we can write $Y_{t} \mid \pi \sim$ $\operatorname{MN}(\pi)$ to represent the multinomial distribution for $Y_{t}^{\prime} s$ realized value without further loss of generality.

How participants might learn about these probabilities is suggested by a direct application of the Bayes' rule. Suppose a player starts with some prior beliefs on the distribution over sales $\pi \sim F_{0}(\pi)$. As the player learns about the movie, its plot synopsis, directors, actors, budget, and other information, they will update their beliefs about this distribution. Indexing a representative player by $n$ and denoting this acquired and perceived information by $s_{n}(\pi)$, we can represent player $n$ 's beliefs given this information by the conditional distribution over probabilities $\pi \mid s_{n} \sim F_{n}(\pi)$. Taking the conditional expectation of these probabilities, player $n$ 's expectation for the probability distribution over states can be

denoted by $\bar{\pi}_{n}=E\left[\pi \mid s_{n}\right]$. Given these expected probabilities, player $n$ 's expected value can be denoted $\bar{Y}_{n}=E\left[Y \mid \pi=\bar{\pi}_{n}\right]=\sum_{k=1}^{K} \bar{\pi}_{n k} X_{k}$.

Now, suppose players can participate in unfettered communication, sharing their information freely and openly without distortionary incentives. Such communication could in principle allow them to pool all their signals. If there are $N$ players, then the conditional distribution over probabilities can be written $\pi \mid s_{1}, \ldots, s_{N} \sim F_{*}(\pi)$ and the expected probability distribution can be represented by $\bar{\pi}_{*}=E\left[\pi \mid s_{1}, \ldots s_{N}\right]$. This final distribution represents all 
information available to participants regarding the potential values of box office sales and given the information available the aggregated expectation $\bar{Y}_{*}=E\left[Y \mid \pi=\bar{\pi}_{*}\right]=\sum_{k=1}^{K} \bar{\pi}_{* k} X_{k}$ represents the best forecast for possible box office revenues. As we will see in the next section, this distribution and aggregated expectation play a central role in defining the ex-post equilibrium of BOP.

We define two different aspects of information aggregation corresponding to the different features of uncertainty captured by the BOP and GOG mechanisms. A mechanism aggregates distributional information if the mechanism generates a signal relating to the probabilities $\pi$ that matches $\bar{\pi}_{*}$. Further, mechanism aggregates expectation information if the mechanism generates a forecast of sales that matches $\bar{Y}_{*}$ As the BOP is designed to reflect the different probabilities of different outcomes, we can evaluate the degree to which it successfully aggregates distributional information as well as expectation information. The GOG mechanism is specifically focused on deriving a point estimate that characterizes expectations, so our tests focus primarily on whether the GOG aggregates expectation information.

Definition: Suppose $Y \mid \pi \sim M N(\pi)$ and suppose players $1, \ldots, N$ privately observe signals $s_{1}, \ldots, s_{N}$ that are informative about $\pi$ and, consequently, about $Y$ as well. Let $\bar{\pi}_{*}=E\left[\pi \mid s_{1}, \ldots s_{N}\right]$ and $\bar{Y}_{*}=\sum_{k=1}^{K} \bar{\pi}_{*, k} x_{k}$ represent the conditional expected probabilities of each state and conditional expectation for $Y$ given all information available to participants.

- A mechanism $G$ that generates a forecast $\hat{\pi}_{G}$ for $\pi$ aggregates distributional information if $\hat{\pi}_{G}=\bar{\pi}_{*}$.

- If the mechanism $G$ generates a forecast $\hat{Y}_{G}$ for $Y$, that forecast aggregates expectation information if $\hat{Y}_{G}=\bar{Y}_{*}$.

In a laboratory environment, $\bar{\pi}_{*}$ is known by virtue of experimental controls and is thus available to researchers when testing the underlying model. However, in field environments, such as box office prediction, only the realized sales become available and only after the box office becomes known. By hypothesis, the best available information about the box office can be no better than the actual box office, which becomes the substance of tests and evaluations. 
We discuss these tests after presenting a summary of the data in the next section, now turning to consider how the mechanisms' incentives guide participants' behavior so that information aggregation can be achieved.

\section{B. Incentives, Behavior, and Information Aggregation in Box Office Prophecy}

The BOP mechanism shares many features with the Information Aggregation Mechanisms studied experimentally in Plott, Wit, and Yang (2003) and Axelrod, et al. (2009). The mechanism is closest to that studied in a field implementation forecasting revenue at Intel by Gillen, Plott, and Shum (2015), which presents a general model that heavily influenced the discussion here. This model is used to characterize key features of the BOP's incentives and how they support distributional information aggregation. The model hinges on the incentives that encourage individuals to demonstrate their differential information in their ticket placements within the BOP. Through these incentives, private information becomes publicly revealed, with the mechanism providing a device for incorporating information into the information that has already been publicly revealed.

To characterize the BOP's state at instance $t$, suppose each bucket $k$ has $\eta_{k}^{(t)}$ tickets in it, allowing $\eta^{(t)}$ to denote the state vector of tickets across all buckets in the BOP. Suppose player $n$ 's interim posterior at time $t$ after conditioning on the BOP's state and his private information, is characterized by expected bucket probabilities $p_{n}^{(t)}=\left[p_{n, 1}^{(t)}, \ldots, p_{n, K}^{(t)}\right]$. Abstracting from beliefs about future behavior, player $n$ 's expected payoff to placing an additional ticket in bucket $x_{k}$ would simply be his posterior expected probability for the realized outcome falling in that bucket divided by the number of tickets already placed in the bucket:

$$
V_{n}^{(t)}\left(x_{k} \mid s_{n}, \eta^{(t)}\right)=\frac{p_{n, k}^{(t)}}{1+\eta_{k}^{(t)}}
$$


If player $n$ is a risk neutral, and an expected utility maximizing agent, then he would place his ticket in the bucket that has the best "odds," i.e., the bucket with the largest posterior likelihood $p_{n, k}$ relative to the number of tickets placed in that bucket $\left(1+\eta_{k}^{(t)}\right)$.

Now, suppose player $n$ has already placed $v_{n, k}$ tickets in bucket $x_{k}$ while continuing to myopically ingore beliefs and considerations about future behavior. Accounting for previous placements by player $n$ and others, the marginal expected payoff from placing an additional ticket in bucket $x_{k}$ is $V_{n}\left(x_{k} \mid v_{n}, s_{n}, \eta\right)=p_{n, k}\left(\frac{1+v_{n, k}}{1+\eta_{k}}-\frac{v_{n, k}}{\eta_{k}}\right)$.

Now, notice that the placement is on the subjective most likely bucket and is thus information revealing only if $v_{n, k} / \eta_{k}$ is small. The property demonstrates that the capacity of ticket placment to reveal information is dependent on the path of the dynamic process of individual ticket placement.

Given these complex dynamics, it is tempting to conclude that information aggregation is not possible in the absence of a convincing theory of the dynamic path, so that the most conclusive theory to hope for would be an impossibilitly result. However, both our theoretical and empirical results demonstrate that this pessimism is not justified.

An expedient way to abstract from dynamics and ensure that the BOP mechanism doesn't distort incentives to report is to consider ex-post equilibria. A strategy profile for player $n$ can be represented by $v_{n}=\left[v_{n, 1}, \ldots, v_{n, K}\right]^{\prime}$, the number of tickets placed in each of the $K$ buckets. Following Krishna (2010)'s textbook definition of an ex-post equilibrium, agents' strategies must represent best responses when evaluated after conditioning on all private signals. The beliefs implied by this information set conveniently matches the aggregated distributional information, $\bar{\pi}_{*}=E\left[\pi \mid s_{1}, \ldots, s_{N}\right]$ introduced in the previous subsection. An important consequence of ex-post equilibrium is that a consensus emerges in the sense that all agents have the same belief about the likelihood of different states. Further, for a strategy 
profile to represent an ex-post equilibrium, all agent must have no incentive to change their own ticket placements given the placement of others, so that:

$$
V\left(v_{n} \mid s_{1}, \ldots, s_{N}, v_{1}, \ldots, v_{N}\right) \geq V\left(v_{n}^{\prime} \mid s_{1}, \ldots, s_{N}, v_{1}, \ldots, v_{N}\right), \forall v_{n}^{\prime} \in \Delta_{K-1}
$$

The theoretical concern, then, is whether or not the mechanism and its implementation introduce incentives leading participants to distort the aggregated distributional information. Our next says that the answer is "no" by characterizing the unique ex-post equilibrium for the mechanism and demonstrating that information aggregates in this equilibrium.

Theorem. Maintaining the assumptions used to define information aggregation, suppose all players follow the symmetric strategy of placing tickets so that $v_{n} \propto \bar{\pi}_{*}$. Then:

a) This outcome represents an ex-post Nash Equilibrium of the BOP Mechanism.

b) This outcome is the unique ex-post Nash Equilibrium of the mechanism.

c) The distribution over tickets for the BOP mechanism under the ex-post equilibrium aggregates distributional information about box office sales.

Proof. We begin by demonstrating that the proposed strategy profile constitutes an ex-post Nash equilibrium. Suppose $v_{i} \propto \bar{\pi}_{*}$ for all $i$ except $n$. Given the information available about expected bucket probabilities and other players' ticket placements, it is optimal for player $n$ to also place their tickets proportionally to the expected bucket probabilities. This partialequilibrium result establishes that $v_{n} \propto \bar{\pi}_{*}$ is a best response for all players.

(a) Consider the decision problem faced by the $n$-th player, conditioning on the players' beliefs $\tilde{p}_{n, k}=\bar{\pi}_{*, k}$ and the assumption that all other players are placing their tickets proportionally to the aggregate posterior beliefs. Player $n$ 's payoff from any ticket allocation is: $E\left[u_{n}(v) \mid s_{1}, \ldots, s_{N}, \alpha\right]=\sum_{k=1}^{K} \frac{v_{n, k}}{(N-1) \bar{\pi}_{*, k}+v_{n, k}} E\left[\pi_{k} \mid s_{1}, \ldots, s_{N}, \alpha\right]=\sum_{k=1}^{K} \frac{v_{n, k}}{(N-1) \bar{\pi}_{*, k}+v_{n, k}} \bar{\pi}_{*, k}$ Taking first order conditions of the Lagrangian that incorporates a shadow cost $(\lambda)$ for the constraint that tickets be fully allocated:

$$
\frac{\partial}{\partial v_{n, k}} E\left[u_{n}(v) \mid s_{1}, \ldots, s_{N}, \alpha\right]=\frac{(N-1) \bar{\pi}_{*, k}^{2}}{\left((N-1) \bar{\pi}_{*, k}+v_{n, k}\right)^{2}}-\lambda=0
$$




$$
\sum_{k=1}^{K} v_{n, k}=1
$$

The budget constraint enforces these first order conditions to balance across each of the $K$ buckets, so player $n$ 's utility maximizing strategy accords with the equilibrium prediction that the players allocate tickets according to the posterior expected bucket probabilities.

$$
\frac{(N-1) \bar{\pi}_{*, k}^{2}}{\left((N-1) \bar{\pi}_{*, k}+v_{n, k}\right)^{2}}=\frac{(N-1) \bar{\pi}_{*, k}^{2}}{\left((N-1) \bar{\pi}_{*, j}+v_{n, j}\right)^{2}} \frac{v_{n, k}}{v_{n, j}}=\frac{\bar{\pi}_{*, k}}{\bar{\pi}_{*, j}}
$$

(b) We now establish uniqueness of the equilibrium outcome. First, we show that at least one player has a profitable deviation if the IAM's distribution of tickets is not proportional to the agreed-upon posterior odds. Second, we show that asymmetric ticket allocations are not supportable with agreement.

(i) Denote the IAM's distribution of tickets over buckets by $\eta$ and suppose $\eta$ is not proportional to $\bar{\pi}_{*}$, then at least one player has a profitable deviation.

Without loss of generality, suppose $\bar{\pi}_{*, 1}>\eta_{1}$ and order the indices so that $\frac{\bar{\pi}_{*, 1}}{\eta_{1}} \geq \frac{\bar{\pi}_{*, 2}}{\eta_{2}} \geq \cdots \geq \frac{\bar{\pi}_{*, K}}{\eta_{K}}$. Choose as player 1 a subject that weakly underallocates tickets to bucket 1 , so that $v_{1,1} \leq \eta_{1}<\bar{\pi}_{*, 1}$ and select bucket $k$ so that $v_{1, k} \geq \eta_{k}$. Consider the gains and losses to player 1 from shifting $\varepsilon$ tickets from bucket $k$ to bucket 1 .

$$
\begin{gathered}
\text { Gains from increasing } v_{1,1}: \quad\left(\frac{v_{1,1}+\varepsilon}{N \eta_{1}+\varepsilon}-\frac{v_{1,1}}{N \eta_{1}}\right) \bar{\pi}_{*, 1}=\frac{N \eta_{1}-v_{1,1}}{N \eta_{1}+\varepsilon} \frac{\bar{\pi}_{*, 1}}{\eta_{1}} \frac{\varepsilon}{N} \\
\text { Cost of decreasing } v_{1, k}: \quad\left(\frac{v_{1, k}-\varepsilon}{N \eta_{k}-\varepsilon}-\frac{v_{1, k}}{N \eta_{k}}\right) \bar{\pi}_{*, k}=\frac{N \eta_{k}-v_{1, k}}{N \eta_{k}-\varepsilon} \frac{\bar{\pi}_{*, k}}{\eta_{k}} \frac{\varepsilon}{N}
\end{gathered}
$$

We want to show that this deviation is profitable for some $\varepsilon>0$, for which it will be sufficient to show:

$$
\frac{N \eta_{1}-v_{1,1}}{N \eta_{1}} \frac{\bar{\pi}_{*, 1}}{\eta_{1}}=\left(1-\frac{v_{1,1}}{N \eta_{1}}\right) \frac{\bar{\pi}_{*, 1}}{\eta_{1}}>\left(1-\frac{v_{1, k}}{N \eta_{k}}\right) \frac{\bar{\pi}_{*, k}}{\eta_{k}}=\frac{N \eta_{k}-v_{1, k}}{N \eta_{k}} \frac{\bar{\pi}_{*, k}}{\eta_{k}}
$$

This inequality holds by the assumptions of our construction: 


$$
\frac{\tilde{p}_{1}}{\eta_{1}}-\frac{\bar{\pi}_{*, k}}{\eta_{k}} \geq \frac{\tilde{v}_{1,1}}{\eta_{1}} \frac{\bar{\pi}_{*, 1}}{\eta_{1}}-\underbrace{\frac{\tilde{v}_{1, k}}{\eta_{k}}}_{\leq 1} \frac{\bar{\pi}_{*, 1}}{\eta_{1}} \frac{\bar{\pi}_{*, k}}{\eta_{k}} \frac{\bar{\pi}_{*, 1}}{\eta_{1}}-\frac{\bar{\pi}_{*, k}}{\eta_{k}}>\frac{1}{N}\left(\frac{\tilde{v}_{1,1}}{\eta_{1}} \frac{\bar{\pi}_{*, 1}}{\eta_{1}}-\frac{\tilde{v}_{1, k}}{\eta_{k}} \frac{\bar{\pi}_{*, k}}{\eta_{k}}\right)
$$

(ii) Suppose the IAM's distribution of tickets is proportional to $\bar{\pi}_{*}$, so that $\frac{\bar{\pi}_{*, 1}}{\eta_{1}}=\frac{\bar{\pi}_{*, 2}}{\eta_{2}}=\cdots=\frac{\bar{\pi}_{*, K}}{\eta_{K}}$, but two players are not playing the same strategy. At least one player has a profitable deviation.

Suppose player 1's allocation differs from the IAM odds. Let $v_{1,1}=\eta_{1}-\xi, v_{1,2}=\eta_{2}+\xi$, and consider the gains and losses to player 1 from shifting $\varepsilon=\xi / N$ tickets from bucket 2 to bucket 1.

$$
\begin{aligned}
& \text { Gains from increasing } v_{1,1}: \frac{N \eta_{1}-v_{1,1}}{N \eta_{1}+\varepsilon} \frac{\varepsilon}{N} \\
& \text { Cost of decreasing } v_{1,2}: \quad \frac{N \eta_{2}-v_{1,2}}{N \eta_{2}-\varepsilon} \frac{\varepsilon}{N}
\end{aligned}
$$

We will show this deviation is profitable by verifying that:

$$
\frac{N \eta_{1}-v_{1,1}}{N \eta_{1}+\varepsilon}>\frac{N \eta_{2}-v_{1,2}}{N \eta_{2}-\varepsilon}
$$

This inequality can be established by direct substitution:

$$
\frac{N \eta_{1}-v_{1,1}}{N \eta_{1}+\varepsilon}=\frac{(N-1) \eta_{1}+\xi}{N \eta_{1}+\xi / N} ; \quad \frac{N \eta_{2}-v_{1,2}}{N \eta_{2}-\varepsilon}=\frac{(N-1) \eta_{2}-\xi}{N \eta_{1}-\xi / N}
$$

Then:

$$
\frac{(N-1) \eta_{1}+\xi}{(N-1) \eta_{2}-\xi}>\frac{N \eta_{1}+\xi}{N \eta_{2}-\xi}>\frac{N \eta_{1}+\xi / N}{N \eta_{2}-\xi / N} \frac{N \eta_{1}-v_{1,1}}{N \eta_{1}+\varepsilon}>\frac{(N-1) \eta_{2}-\xi}{N \eta_{1}-\xi / N}
$$

(c) By the definition of $\bar{\pi}_{*}$ and the results of Parts (a) and (b), the IAM ticket allocation represents rational expectations for $E\left[\pi \mid s_{1}, \ldots, s_{N}, \alpha\right]$. Clearly, if every player places tickets proportionally to $\bar{\pi}_{*}$, then the aggregated distribution of tickets in the IAM will match this distribution. Distributional information aggregation is thus established. 
The theory identifies the possibility of information aggregation. It also suggests the possibilities of information aggregation failure that be result from the difficulties of operation, complexity, dynamics of adjustment, the existence of multiple equilibria and asymmetric equilibria. Thus, information aggregation is an empirical issue. In the next section we explore the empirical evidence.

\section{C. The Possibility of Expectational Information Aggregation in Guess of Guesses}

The Guess of Guesses (GOG) mechanism is analyzed from the perspective of player 1 without loss of generality. Consider ex-post deviations in the mechanism, allowing player 1 to revise her guess after observing the choices of all other players in GOG. Clearly, she would be able to compute the median of those guesses and identify either a new guess that would be the unique median or pool with the other players submitting the median guess. Extending this logic, ex-post equilibrium restricts all players to submit identical guesses while placing no limitation on what the coordinated guess must be. Given the single-shot nature of the mechanism, however, such coordination is clearly implausible. Consequently, we need to consider how player's form beliefs about other players' guesses and how they react to those beliefs in reporting their own guess.

The issue is resolved by the observation that if a player believes that the other's beliefs are the same as their own beliefs, in which case the optimal strategy is to report that belief. This assumption is supported by the law of iterated expectations, since the coarsened expectation of a more refined conditional expectation is simply the coarsened

expectation. More precisely, for any random variable $Z, E\left[E\left[Z \mid s_{1}, \ldots, s_{n}\right] \mid s_{i}\right]=E\left[Z \mid s_{i}\right]$, so that when accounting for uncertainty in the distribution over box office revenues when taking the conditional expectation:

$$
\hat{Y}_{n}=E\left[E\left[E[Y \mid \pi] \mid s_{1}, \ldots, s_{N}\right] \mid s_{n}\right]=E\left[E[Y \mid \pi] \mid s_{n}\right]=\sum_{k=1}^{K} \bar{\pi}_{n, k} X_{k}
$$

Applying this result to the problem of guessing the median forecast requires assuming symmetry for the distribution over sales. Under symmetry, the median will equal the 
expectation and so we can apply the Law of Iterated Expectations directly to the conditional median.

We close this section by again noting that information aggregation within the GOG mechanism is not a necessary outcome, but simply a possible outcome. The degree to which information aggregates in practice using either the BOP or the GOG mechanism is an empirical question. We do note, however, that information contained in GOG must reflect the private information held by individuals prior to the implementation of BOP. Our discussion here simply motivates some of the economic reasoning supporting this possibility as a means of motivating the empirical analysis in the next section.

\section{Results: Mechanism Performance}

This section considers the empirical evidence from Boxoffice Prophecy (BOP) and Guess of Guesses (GOG) to test the degree to which the two mechanisms aggregate information and elicit beliefs. We begin by analyzing forecasts derived from each of the mechanisms and the information content of these forecasts indicates that both mechanisms effectively aggregate expectation information. This result indicates both that our subjects have information about the box office potential for a given movie and that the mechanisms can collect that information. Since the BOP reveals greater detail about participants' beliefs, we can also investigate the degree to which the BOP aggregates distributional information. We find the aggregated distribution reported by the BOP accurately matches the likelihood of different outcomes. We close this section by returning to the GOG mechanism and, by analyzing the distribution over guesses, suggest the mechanism is eliciting a measure of central tendency for each participant's beliefs.

\section{A. Expectation Information Aggregation in BOP and GOG}

If a mechanism aggregates expectation information, then measures of those expectations from the mechanism should present rational forecasts for realized sales. As the first step in investigating expectation information aggregation, we define natural forecasts for the two mechanisms. The mean BOP forecast is calculated as the mean of the forecast 
distribution for each trial of the BOP mechanism. ${ }^{6}$ The GOG forecast is calculated as the average guess reported in the guess of the median guess mechanism. These forecasts could be defined differently, such as using medians or modes from the mechanisms' reports, but these alternative formulations give qualitatively similar results.

\section{A.1 Summary Statistics and Forecast Accuracy}

Table 3 presents summary box office statistics for the movies we studied, along with statistics describing the two mean forecasts. ${ }^{7}$ In the sample for all movies, the BOP forecasts are slightly optimistic, with an average box office forecast of AU\$200k above the actual box office. The root mean square forecast error (RMSFE) provides a good first-look at forecast accuracy. Comparing the forecasts' RMSFE to the standard deviation of box office revenues indicates the degree to which the mechanisms improve upon the ex-post average as a benchmark forecast. These forecasts perform reasonably well with a root mean square forecast error (RMSFE) of AU\$2m compared to a cross-sectional standard deviation of AU\$7m, the latter of which corresponds to the RMSFE of using the ex post sample average as a forecast. The forecast presents an especially significant improvement over the ex-post average for Blockbuster movies, which have substantially greater variability in revenue.

\footnotetext{
${ }^{6}$ We compute the Mean BOP Forecast with a weighted average of the value assigned to each bin, weighted by the number of tickets in that bin. Specifically, if the $k^{\text {th }}$ bin pays off if revenues are between $x_{k-1}$ and $x_{k}$, we assign bin $k$ the value of $v_{k}=\left(x_{k-1}+x_{k}\right) / 2$. Since bins are equally-spaced, we assign the first bin a value of $\left(x_{1}+\left(x_{1}-\left(x_{2}-x_{1}\right)\right)\right) / 2$ and treat the last bin symmetrically. There are several different ways to label these extreme bins and our results are robust to reasonable treatments (obviously, labeling the last bin as having an infinite value would be problematic). Given these defined values, if the $k^{\text {th }}$ bin has $\eta_{k}$ tickets in it, then the Mean BOP Forecast is simply:

$$
\text { BOP Mean }=\frac{1}{\sum_{k=1}^{K} \eta_{k}} \sum_{k=1}^{k} \eta_{k} v_{k}
$$

${ }^{7}$ We drop 10 observations due to issues related to censoring. For example, Art House films that ended up in the lowest bucket when the buckets were designed too large ex-ante. Dropped titles are noted in Appendix A table.
} 
Table 3. Summary Statistics for Box Office Sales and Mechanism Forecasts

\begin{tabular}{|c|c|c|c|c|c|c|}
\hline & \multicolumn{4}{|c|}{ Panel A: Boxoffice Prophecy } & \multicolumn{2}{|c|}{ Panel B: Guess of Guesses } \\
\hline & All Data & Art House & Regular & Blockbuster & & \\
\hline No. of Markets & 74 & 27 & 29 & 18 & & \\
\hline No. of Movies & 53 & 19 & 19 & 15 & No. of Movies & 41 \\
\hline \multicolumn{5}{|l|}{ Box Office Sales } & \multicolumn{2}{|c|}{ Box Office Sales } \\
\hline Average & 4,320 & 669 & 3,374 & 11,322 & Average & 10,553 \\
\hline Std Dev & 7,154 & 1,614 & 3,271 & 11,114 & Std Dev & 13,337 \\
\hline \multicolumn{5}{|c|}{ Boxoffice Prophecy Mean Forecast } & \multicolumn{2}{|c|}{ Guess of Guesses Forecast } \\
\hline Average & 4,523 & 980 & 3,743 & 11,092 & Average & 8,790 \\
\hline Std Dev & 6,728 & 1,913 & 3,059 & 10,329 & Std Dev & 10,082 \\
\hline RMSFE & 2,152 & 992 & 2,042 & 3,293 & RMSFE & 7,556 \\
\hline
\end{tabular}

Notes: This table presents summary statistics for the movies forecasted using the Boxoffice Prophecy (Panel A) and Guess of Guesses (Panel B) Mechanisms. Reported sales and forecast statistics are in units of AUD1,000. Box office Average and Std Dev are calculated across movies in each of the subsamples. The Boxoffice Prophecy Mean Forecast corresponds to the mean of the forecast distribution from the Boxoffice Prophecy mechanism, with Average, Std Dev, and Root Mean Square Forecast Error (RMSFE) calculated across markets. The Guess of Guesses Forecast corresponds to the average reported guess in the Guess of Guesses mechanism.

\section{A.2 Forecast Rationality and Robustness Tests}

To provide a more refined perspective of the information content of the mechanisms' forecasts, we apply standard tests for forecast evaluation. Mincer-Zarnowitz regressions evaluate the scale and bias of a forecast by regressing the realized outcome $\left(Y_{t}\right)$ on a constant and the forecast $\left(\hat{Y}_{t}\right)$ :

$$
Y_{t}=\alpha+\beta \hat{Y}_{t}+\varepsilon_{t}
$$

An unbiased forecast will have $\alpha=0$ and a forecast with accurate scaling would have $\beta=1$. Each of these hypotheses can be evaluated individually using a $t$-test and, jointly, using an $F$ test. Figure 3 presents the results of these forecast tests for opening weekend revenues beneath scatter plots corresponding to the fitted-line regressions. 


\section{Figure 3. Forecast Line Fits and Rationality Tests}

Panel A: BOP Mechanism

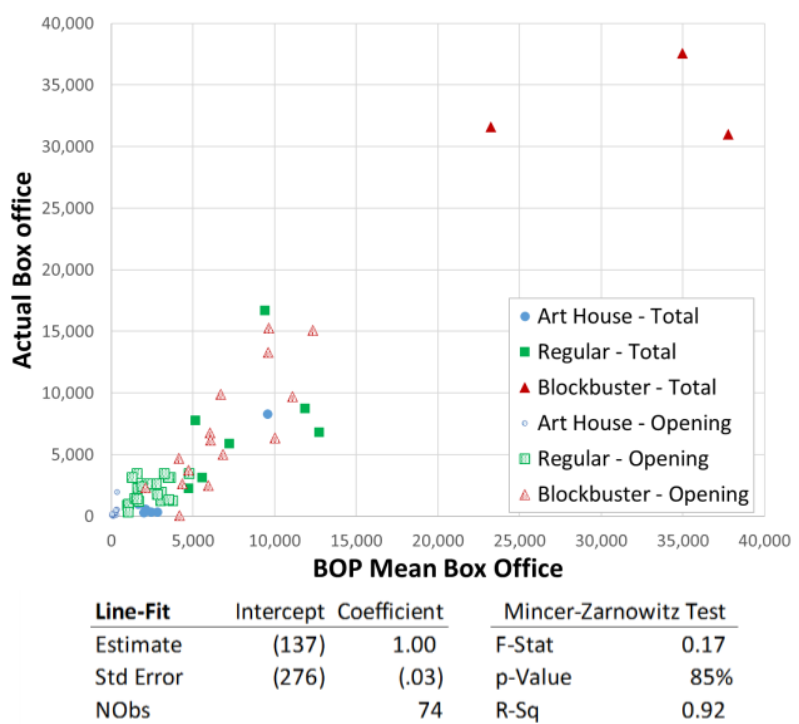

Panel B: GOG Mechanism

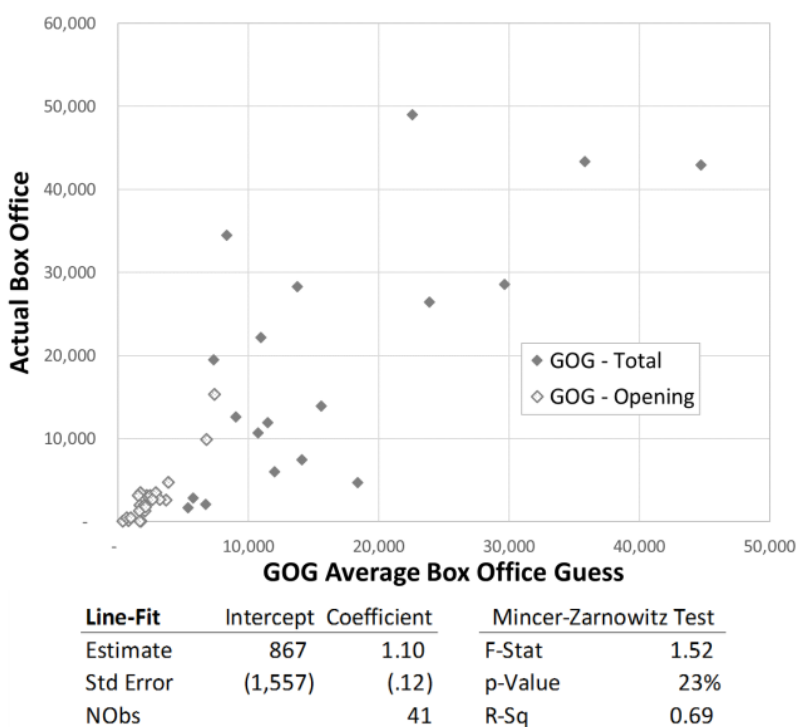

Notes: This figure presents Mincer-Zarnowitz regressions (Equation ${ }^{* *}$ ) and forecast rationality tests using the Boxoffice Prophecy (Panel A) and Guess of Guesses (Panel B) Mechanisms. The Boxoffice Prophecy Mean Forecast corresponds to the mean of the forecast distribution from the Boxoffice Prophecy mechanism and the Guess of Guesses Forecast corresponds to the average reported guess in the Guess of Guesses mechanism.

To evaluate the robustness of these results, we analyze the forecast accuracy in some subsamples of the BOP data, slicing according to the type of movie for which revenues are being forecast. The summary statistics for each subsample appear in Table 3 and the MincerZarnowitz regressions, along with the corresponding scatter-plots, appear in Figure 4. These results illustrate the robustness of information aggregation across the different movie markets, indicating that the BOP mean performs quite well with regular movies and blockbusters, but underperform in forecasting opening box office for art house movies. The latter performance breakdown is driven by two artifacts that made these movies particularly difficult to forecast. First, there is relatively little variability in the revenues for our sample of art house movies. Consequently, the simple average revenue for art house movies in general is a good forecast for any single art house movie. Second, the buckets used for BOP in the art house movies may 
have been too large. In some of these movies, the smallest bucket was AU\$250k, censoring the forecasts for movies that had an average revenue of AU\$400k.

Figure 4. Forecast Rationality Tests in Subsamples
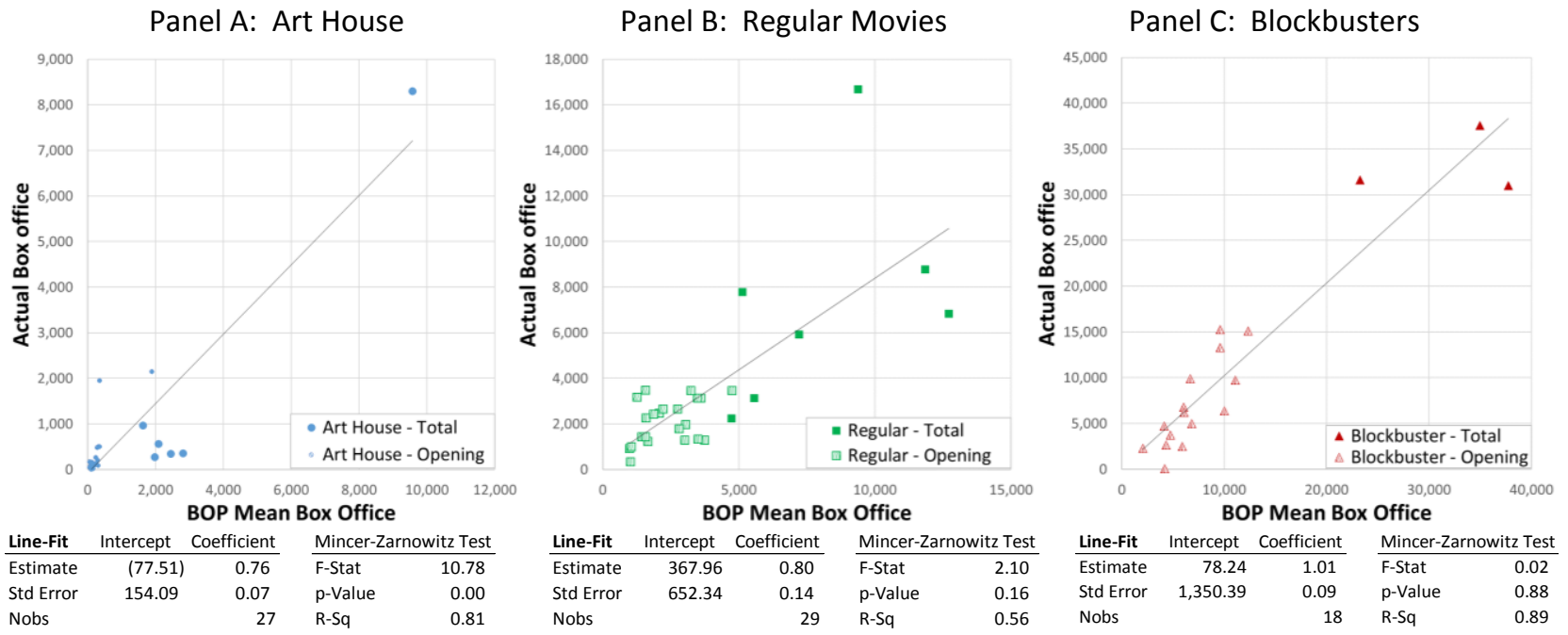

Notes: This figure presents Mincer-Zarnowitz regressions (Equation ${ }^{* *}$ ) and forecast rationality tests for subsamples of the Boxoffice Prophecy Mechanism. The Boxoffice Prophecy Mean Forecast corresponds to the mean of the forecast distribution from the Boxoffice Prophecy mechanism. The category of a movie was determined by the researchers prior to operation of the mechanism.

\section{A.3 Comparing BOP and GOG Forecast Accuracy}

In looking at the properties of the GOG forecasts, we first note that the average box office for movies in this mechanism is much higher than the average in the BOP. This selection of more blockbuster-like movies into the GOG tests is driven by the long-term nature of the GOG mechanism. Movies that have six months of lead time before their opening tend to be larger productions requiring more intensive production and marketing efforts. The forecasts themselves tended to have a bit more of a negative bias, understating average box office revenues by about AU\$1.75M. However, despite this bias, the RMSFE for the GOG forecasts represents a significant improvement over the unconditional standard deviation of box office themselves. As such, the GOG forecasts do reflect substantial information about potential box office revenues. 
For a subsample of the movies in the 2010 iteration, we have concurrent forecasts available from both the BOP and GOG mechanisms. Within this subsample, we can directly test the accuracy of the forecasts using Diebold-Mariano (1991) tests. These are simple $t$-tests that evaluate whether the mean forecast loss from the BOP forecast is lower than the mean forecast loss from the GOG forecast. Specifically, for each movie and forecast mechanism, we define the forecast loss using squared error:

$$
l_{t}^{(k)}=\left(Y_{t}-\hat{Y}_{t}^{(k)}\right)^{2}, k \in\{\mathrm{BOP}, \mathrm{GOG}\}
$$

With this forecast loss, we can calculate the difference in forecast loss between the two mechanisms as:

$$
\delta_{t}=l_{t}^{(\mathrm{BOP})}-l_{t}^{(\mathrm{GOG})}
$$

If BOP and GOG are equally accurate, the expected difference in loss between the two mechanisms would be zero. Consequently, we can use a $t$-test to evaluate this hypothesis, $E\left[\delta_{t}\right]=0$, against the alternative that BOP is more (less) accurate than GOG, $E\left[\delta_{t}\right]<0$ $\left(E\left[\delta_{t}\right]>0\right)$. The results of the Diebold-Mariano tests appear in Table 4, Panel A. Despite BOP delivering a more accurate forecast for $59 \%$ of movies, the small sample of only 23 forecasts is sufficiently small and variable to prevent this difference in loss from reaching statistical significance.

Given the inconclusive results from the Diebold-Mariano tests, we might wish to consider the information content of the two forecasts. Specifically, if we were to combine the BOP and GOG forecasts, how much weight would we assign to the BOP forecast and how much would we assign to GOG? These weights can be easily calculated from the Fair-Shiller regressions:

$$
Y_{t}=\alpha+\omega_{B O P} \hat{Y}_{t}^{(B O P)}+\omega_{G O G} \hat{Y}_{t}^{(G O G)}+u_{t}
$$

The results reported in Table 4, Panel B, indicate that significant weight in a combined forecast is assigned to the both forecast mechanisms. The weight assigned to BOP is not statistically different from zero and the weight assigned to GOG isn't significantly differentiated from one. These joint restrictions are tested in the Encompassing tests, which are $F$-tests for the joint hypothesis that the weight to one forecast is equal to one while the weight of another 
is equal to zero. We can reject the null hypothesis that the GOG forecast encompasses the BOP forecast (i.e., that $\omega_{G O G}=1 \cap \omega_{B O P}=0$ with a $p$-value of $0 \%$ ) but we cannot reject the hypothesis that BOP encompasses the GOG forecast ( $\omega_{G O G}=0 \cap \omega_{B O P}=1$ with a $p$-value that rounds to $0 \%)$. These results indicate that both mechanisms contain useful information for forecasting actual box office sales.

Table 4. Direct Comparison of Mechanism Forecasts

\begin{tabular}{|c|c|c|c|c|c|c|}
\hline \multicolumn{3}{|c|}{ Panel A: Diebold-Mariana Test } & \multicolumn{4}{|c|}{ Panel B: Fair-Shiller Regression } \\
\hline & & & & Intercept & BOP Weight & GOG Weight \\
\hline No. Obs & & 22 & Estimate & $(1,361)$ & 0.31 & 1.42 \\
\hline BOP Outperfo & Freq. & $59 \%$ & Std. Error & (521) & $(0.28)$ & $(0.36)$ \\
\hline Average Delta & $t$-stat & $p$-value & \multicolumn{2}{|c|}{ Encompassing Tests } & $F(., 1,0)$ & $F(., 1,0)$ \\
\hline \multirow[t]{2}{*}{ (577) } & $(0.21)$ & $84 \%$ & \multirow{2}{*}{\multicolumn{2}{|c|}{$\begin{array}{l}F \text {-stat } \\
p \text {-value }\end{array}$}} & 7.88 & 10.07 \\
\hline & & & & & $0 \%$ & $0 \%$ \\
\hline
\end{tabular}

Notes: This table presents direct tests comparing the accuracy of forecasts derived from the Boxoffice Prophecy and Guess of Guesses Mechanisms. The Boxoffice Prophecy Mean Forecast corresponds to the mean of the forecast distribution from the Boxoffice Prophecy mechanism and the Guess of Guesses Forecast corresponds to the average reported guess in the Guess of Guesses mechanism. Panel A presents statistics for the DieboldMariano test of relative forecast accuracy. Panel B presents the results of the Fair-Shiller regression and two $F$ tests evaluating whether the BOP forecast encompasses the GOG forecast and vice-versa.

These results raise the question of what causes the two mechanisms to generate differential and separately-informative signals about box office revenue. One possibility is that both mechanisms, by asking similar questions, cause subjects to consider different aspects of a movie and its potential box office appeal in reporting their forecasts. Another possibility is that the BOP mechanism, by allowing for interactions among participants, leads to more refined information aggregation than is achieved by the GOG mechanism's inherently private format. This interpretation suggests that the BOP mechanism is able to avoid the "private equilibrium" pitfall that presents theoretical challenges to information aggregation in market mechanisms. 


\section{B. Distributional Information Aggregation in BOP}

If none of our participants have any information at all about how movies will perform, no mechanism for aggregating their information would have any ability to forecast box office revenues. However, the summary statistics in Table 3 indicate that our study participants do have valuable information for forecasting box office sales, particularly in larger movies. The next question we investigate concerns how accurately the BOP recovers and reports this information. To evaluate this question, we translate the realized box office revenues into the quantiles from the BOP forecast distribution. Suppose the proportion of tickets in each bucket $k$ for movie $i^{\prime}$ s BOP is $\eta_{k}$ and denote the realized box office revenues by $Y_{i}$, we compute the BOP quantile, denoted $Q_{i}$, using the formula:

$$
Q_{i}=\sum_{k=1}^{K} 1\left\{x_{k}<Y_{i}\right\} \eta_{k}+\sum_{k=1}^{K} 1\left\{x_{k} \geq Y_{i}>x_{k-1}\right\} \eta_{k} \frac{Y_{i}-x_{k-1}}{x_{k}-x_{k-1}}
$$

If the BOP's distribution over tickets accurately reflects the true uncertainty in the distribution over realized sales, then these quantiles will be uniformly distributed. We can use this result to test the BOP's performance. If either our participants lack information about box office revenues or if the BOP fails to aggregates that information, then a Kolmogorov-Smirnoff test would reject the null hypothesis that these quantiles are drawn from a uniform distribution. 


\section{Figure 5. Kolmogorov-Smirnoff Test of all Films from BOP}

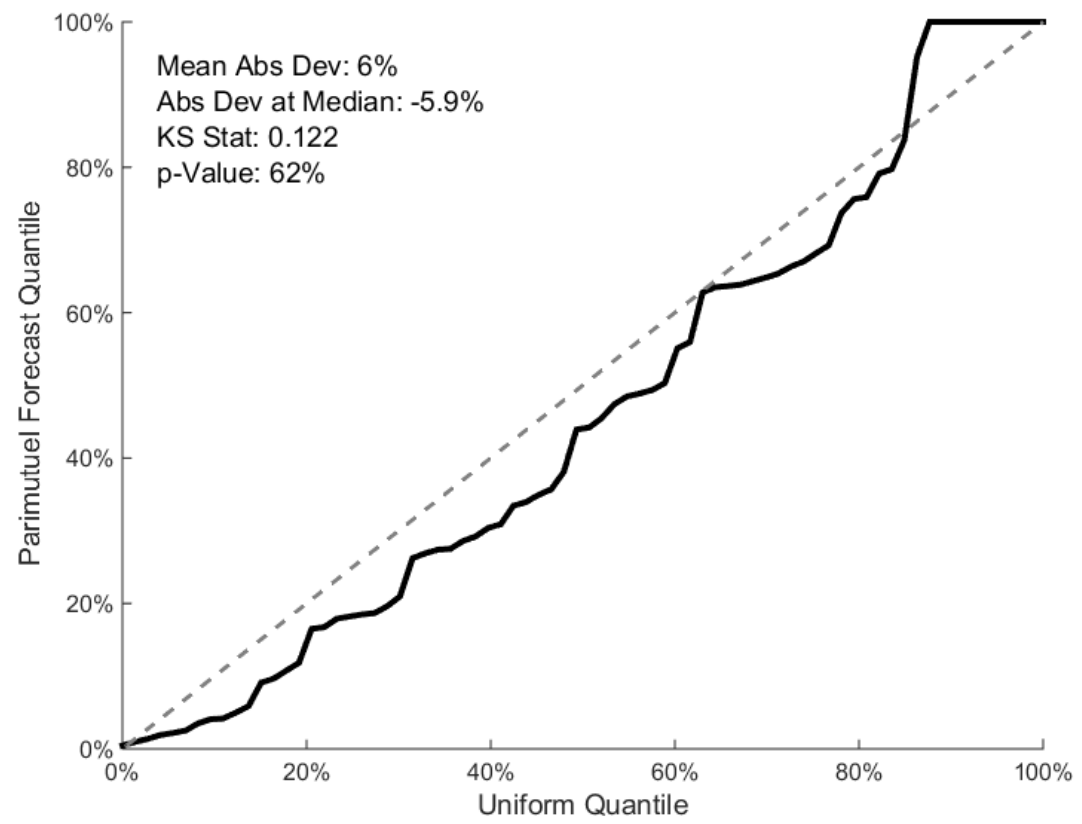

This figure tests the accuracy of the distributions over sales reported by the BOP mechanism.

Figure 5 plots the cumulative distribution function for these quantiles against the CDF for a uniform distribution. The apparent accuracy of the BOP's distribution is truly striking. The CDF very closely tracks the 45-degree line implied by the uniform distribution, with the mean absolute deviation of only $6 \%$. The Kolmogorov-Smirnoff test statistic achieves a $p$-value of $62 \%$, which is strikingly high even though the sample consists of only 74 observations.

In order to explore the robustness of BOP's accuracy in characterizing the distribution of a movie's opening weekend box office revenues, we again turn to the subsamples for forecasts by different movie types. These results are reported in the three panels of Figure 4 . None of these tests indicate the distribution over quantiles is statistically significantly different from the uniform distribution for any of the samples. However, this finding could be driven by a lack of power due to the limited sample sizes in these subsamples. 


\section{Figure 6. Kolmogorov-Smirnoff Test for Subsamples of BOP Films}
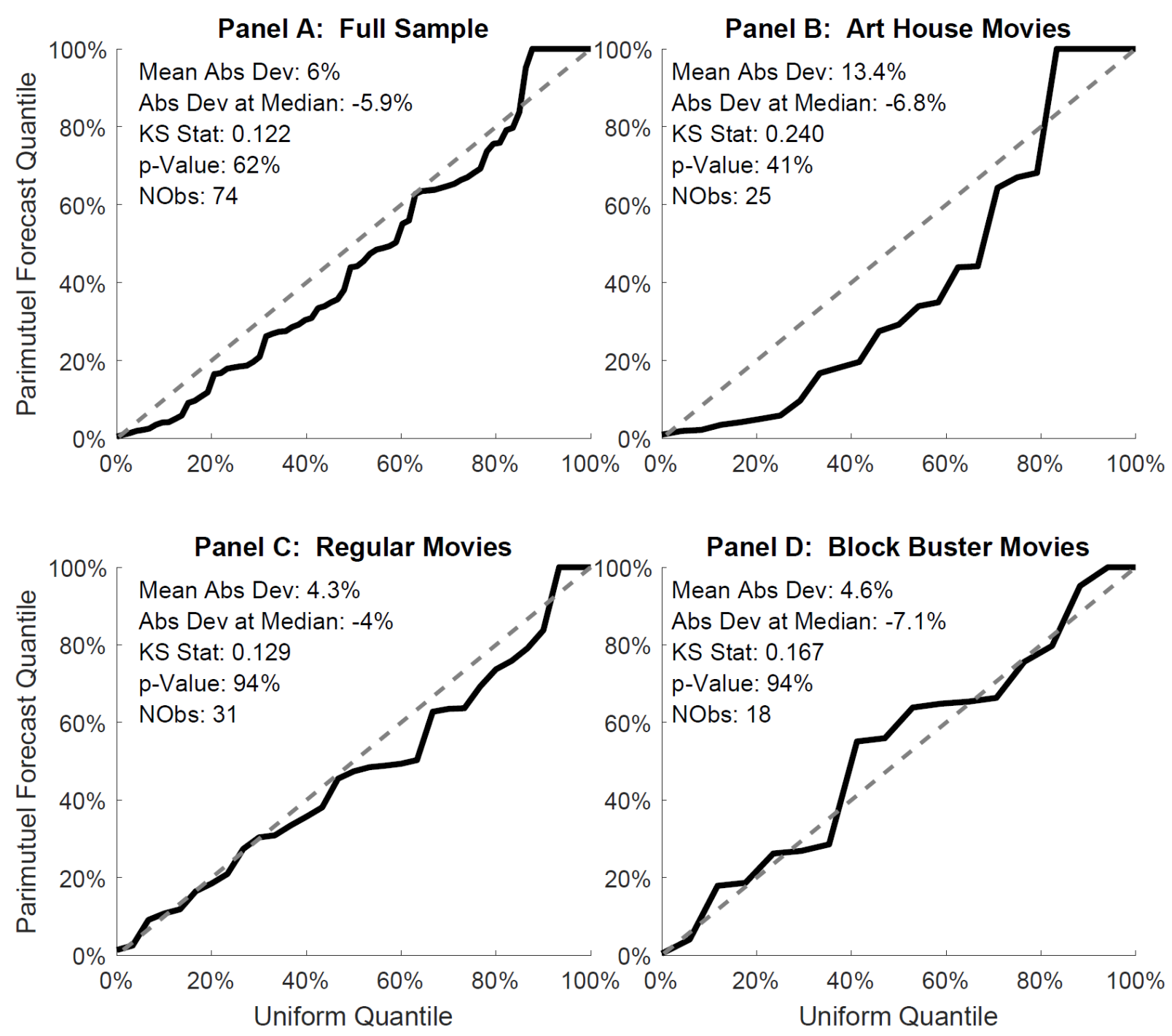

This figure tests the accuracy of the distributions over sales reported by the BOP mechanism for different types of movies.

While the distributions over quantiles for Regular and Blockbuster movies are nearly identical to the uniform, the results for Art House movies appear to be somewhat distorted. The S-shaped distortion in Art House movies is similar to the Reverse Favorite-Longshot Bias observed by Gillen, Plott and Shum (2015) when generating sales forecasts in settings with little information available to participants. ${ }^{8}$ This relationship could indicate that participants had

\footnotetext{
${ }^{8}$ The Long-Shot Bias arises when market odds overstate the likelihood of low-probability events, an issue that has been discussed extensively in the literature (including four chapters in the Handbook of Sports and Lottery Markets (Hausch and Ziemba, 2008). Researchers have also observed the opposite pattern, a Reverse Long-Shot Bias,
} 
relatively poor information available to forecast the box office for Art House movies, likely because such movies have smaller budgets and are often staffed with less well-known film crews and actors.

\section{Conclusions}

The paper focus is the performance of Information Aggregation Mechanisms (IAMs) when applied to forthcoming films in Australia that consist of a variety of different types of films and time frames. Three unknowns form the questions posed for research. Do the intuitions and subjectively held beliefs of a group of film students and industry practitioners contain solid information about the box office of upcoming films? Do either or both of the proposed information aggregations mechanisms (IAMs) collect and organize the beliefs and associated information? How can we know?

The data indicate that the information sought by the mechanisms exists and can be measured. Successful operation is dependent on the information held by participants but a large number of participants is not needed and could be harmful if a large proportion is completely uninformed. The information is not found only in highly technical and experienced sources. Specifically, the intuitions of the students as measured by the parimutuel procedures do contain information about future box office magnitudes and it could be important that this fact was demonstrated to the participants themselves by the early successes of short term predications. The strong correlation between the median of these opinions and the outcome is not an accidental correlation. The conclusion is supported by aggregate distributional information in addition to expectational information. The K-S tests are particularly accurate for regular and blockbuster films while the data from Art House movies appears driven by censoring.

which presents as market odds overstating the likelihood of high-probability events and understating the probability of low-probability events. A number of strategic or behavioral features of prediction markets might drive these phenomena, including risk aversion (Jullien and Salanie, 2000), probability weighting (Snowberg and Wolfers, 2010), heterogeneous beliefs (Gandhi and Serrano-Padial, 2012), and strategic models (Ottaviani and Sorensen, 2010). 
Both the Boxoffice Prophecy (derived from parimutuel betting processes) and the Guess of Guesses (incentivized guess of what others will guess) show promise as information aggregation mechanisms. Support is found the summary statistics regarding RMSFE and in the Mincer-Zarnowitz regressions for forecast accuracy. The data suggest that the BOP provides more accurate and more precise forecasts. In particular, the Diebold-Mariano tests show BOP to be more accurate and encompassing tests indicate the BOP forecast contains information not contained in the GOG even though both contain information not contained in the other. The fact that GOG represents accuracy based only on private information, acquired without the benefit of conversations with others, supports an interpretation of the difference between the GOG and BOP as a consequence of information aggregation. The private information held by individuals and reflected in their GOG behavior as compared to the information subsequently gathered in the BOP process, reflects information aggregation resulting from the application of the BOP.

A more philosophical question rests beneath the practical questions. The mechanisms were developed from incomplete theory tested through laboratory methods. Can the laboratory methods from experimental economics tell us anything about the operations of the naturally occurring phenomena found in field environments? The first challenge rests on a model that that translates the abstract features of the model into the operational concepts required to connect the model to variables found naturally occurring. This connection was provided through a generalized Dirichlet distribution. The approach rests on an assumption that the information about the box office is in the form of a distribution of possible outcomes and that information aggregation should result in a distribution. The model is developed to produce and test a full frequency distribution of box office revenues in addition to testing the accuracy of a prediction of the expected value of box office revenues. The latter is important because some IAMs like the GOG, make only point predictions. The developments allow tests of accuracy of the two IAMs as well as comparisons of their relative accuracy.

The second challenge focused on whether or not the economic principles uncovered in laboratory experiments and the procedures that evolved from laboratory experiences are sufficiently robust to justify a degree of trust in them when applied to substantially different 
conditions found outside the laboratory. The paper offers a step in toward meeting both challenges. The theory of how the mechanism works draws from both market theory and game theory but the understanding provided by these theories is incomplete, leaving much open for empirical resolution and a healthy application of "as if" methodologies. The broad empirical relationships suggested do exist and are open invitations for theoretical work. The distribution of box office predicted is close to the actual distribution of box office amounts. Using a KS test the hypotheses that the two distributions are the same, the predicted and the actual, cannot be rejected. This result demonstrates that the information exists in the intuitions of the test group and that it is collected by the IAM. Using the RMSFE the expectations prediction of both the BOP and GOG cannot be rejected as producing an accurate mean of the actual box office amounts. The accuracy is supported for all types of movies and all futures but films classified as Art House are the least predictable and predictability degrades as the distance of the prediction advances in the future. Comparisons of the two IAM reveal that BOP is more accurate than GOG.

Reflections on the underlying methodological and philosophical issues give a clear message. The intuitions of individuals as represented by subjective probabilities do contain solid information. The basic principles of economics are remarkably robust and are usefully captured by laboratory experimental methods.

\section{References}

Asur, S. and Huberman, B. A. (2010), "Predicting the Future with Social Media", Proceedings of the 2010 IEEE/WIC/ACM International Conference on Web Intelligence and Intelligent Agent Technology, 492-499.

Axelrod, B. S., Kulick, B. J., Plott, C. R. and Roust, K. A. (2009), “"The Design of Improved Parimutuel-Type Information Aggregation Mechanisms: Inaccuracies and the Long-Shot Bias as Disequilibrium Phenomena". Journal of Economic Behavior and Organization, 69(2):170-181.

Berg, J., Forsythe, R., Nelson, F. and Rietz, T. (2008), "Results from a Dozen Years of Election Futures Markets Research", Volume 1 of Handbook of Experimental Economics Results, Chapter 80, pages 742-751. Elsevier.

Chen, K. Y. and Plott, C. R. (2002), "Information Aggregation Mechanisms: Concept, Design, and Implementation for a Sales Forecasting Problem", Mimeo. 
De Vany, A. S. (2004), Hollywood Economics: How Extreme Uncertainty Shapes the Film Industry, Routledge.

Diebold, F. X. and Mariano R. S. (1995), "Comparing Predictive Accuracy". Journal of Business \& Economic Statistics, 13(3):253-263.

Doshi, L., Krauss, J. Nann, S. and Glor, P. (2010), "Predicting Movie Prices Through Dynamic Social Network Analysis", Procedia Social and Behavioral Sciences, 2:6423-6433.

Eliashberg, J., Elberse, A. and Leenders, M. (2006), "The Motion Picture Industry: Critical Issues in Practice, Current Research, and New Research Directions", Marketing Science, 25(6):638-661.

Foutz, N. and Jank, W. (2010), "The Wisdom of Crowds: Pre-release Forecasting via Functional Shape Analysis of the Online Virtual Stock Market", Marketing Science, 29(3):568-579.

Fair, R. C. and Shiller, R. J. (1990), "Comparing Information in Forecasts from Econometric Models", American Economic Review, 80(3):375-89.

Gandhi, A. and Serrano-Padial, R. (2012), "Are Beliefs Heterogeneous? The Case of the Longshot Bias". Working Paper..

Gillen, B., Plott, C. R. and Shum, M. (2015), "A Parimutuel-Like Mechanism for Information Aggregation: A Field Test Inside Intel", Journal of Political Economy (forthcoming), California Institute of Technology, Working Paper No. 1367.

Google (2013), “Quantifying Movie Magic with Google Search”, Google Whitepaper: Industry Perspectives + User Insights.

Hadida, A. (2008), "Motion Picture Performance: A Review and Research Agenda", International Journal of Management Reviews, 11(3):297-335.

Hausch, D. B. and Ziemba, W. T. (2008), Handbook of Sports and Lottery Markets. NorthHolland.

Jullien, A. and Salanie, B. (2000), “Estimating Preferences Under Risk: The Case Of Racetrack Bettors", Journal of Political Economy, 108:503-530.

Kalovcova, K. and Ortmann. A. (2009), "Understanding the Plott-Wit-Yang Paradox. CERGE-EI" Working Papers WP397, The Center for Economic Research and Graduate Education - Economic Institute, Prague.

Koessler, F., Noussair, C. and Ziegelmeyer, A. (2012), “Information Aggregation and Belief Elicitation in Experimental Parimutuel Betting Markets", Journal of Economic Behavior and Organization, 83:195-208.

Krishna, V. (2010) , Auction Theory $2^{\text {nd }}$ Edition, Academic Press, San Diego.

McKenzie, J. (2012), "The Economics of Movies: A Literature Survey”, Journal of Economic Surveys, 26(1):42-70.

McKenzie, J. (2013), "Predicting Box Office with and without Markets: Do Internet Users Know Anything?", Information Economics and Policy, 25:70-80. 
Mestyan, M., Yasseri, T. and Kertesz, J. (2013), "Early Prediction of Movie Box Office Success Based on Wikipedia Activity Big Data", PLOS ONE, 8(8):e71226.

Mishne, G. and Glance, N. (2006), "Predicting Movie Sales from Blogger Sentiment", Proceedings of AAAl 2006 Spring Symposium on Computational Approaches to Analyzing Weblogs (AAAI-CAAW), 155-158.

Nagel, R. (1995), "Unraveling in Guessing Games: An Experimental Study", American Economic Review 85 (5):1313-1326.

Ottaviani, M. and Sørensen, P. N. (2010), "Noise, Information, and the FavoriteLongshot Bias in Parimutuel Predictions", American Economic Journal: Microeconomics, 2(1):58-85.

Pennock, D., Lawrence, S., Giles, C. L. and Nielsen, F. A. (2001), "The Real Power of Artificial Markets", Science, 291(5506), 987-988. [A longer version of this article is also available at http://artificialmarkets.com/am/pennock-neci-tr-2000-168/ ]

Plott, C. R. and Roust, K.A. (2009), "The Design Of Information Aggregation Mechanisms: A Two-Stage Parimutuel Market To Avoid Mirages Bubbles", Mimeo.

Plott, C. R. (2000), "Markets as Information Gathering Tools". Southern Economic Journal, 67(1):1-15.

Plott, C. R. and Sunder, S. (1982), "Efficiency of Experimental Security Markets with Insider Information: An Application of Rational-Expectations Models". Journal of Political Economy, 90(4):663-698.

Plott, C. R. and Sunder, S. (1988), "Rational Expectations and the Aggregation of Diverse Information in Laboratory Security Markets". Econometrica, 56(5):10851118.

Plott, C. R., Wit, J. and Yang, W. C. (2003), "Parimutuel Betting Markets As Information Aggregation Devices: Experimental Results". Economic Theory, 22(2):311-351.

Prelec, D. (2004), “A Bayesian Truth Serum for Subjective Data”, Science, 306(5695):462-466.

Snowberg, E. and Wolfers, J. (2010). "Explaining The Favorite-Long Shot Bias: Is It RiskLove Or Misperceptions?” Journal of Political Economy, 118(4):723-746.

Spann, M. and Skiera, B. (2003), "Internet-Based Virtual Stock Markets in Business Forecasting", Management Science, 49(10), 1310-1326.

Weaver, S. and Prelec, D. (2013), "Creating Truth-Telling Incentives with the Bayesian Truth Serum", The Journal of Marketing Research, 1:289-302. 
FOR ONLINE PUBLICATION Appendix A: Table of All Films

\begin{tabular}{|c|c|c|c|c|c|c|c|}
\hline ID & Title & $\begin{array}{l}\text { Experiment } \\
\text { Date }\end{array}$ & $\begin{array}{l}\text { Release } \\
\text { Date }\end{array}$ & $\begin{array}{l}\text { Bucket } \\
\text { Size }\end{array}$ & $\begin{array}{l}\text { Analysis } \\
\text { Definition }\end{array}$ & $\begin{array}{l}\text { BOP } \\
\text { (OD, WE, TOT) }\end{array}$ & $\begin{array}{l}\text { GOG } \\
\text { (WE, TOT) }\end{array}$ \\
\hline 1 & Footy Legends & 28-Jul-06 & 03-Aug-06 & Small & Art-house & WE,TOT & \\
\hline 2 & Miami Vice & 04-Aug-06 & 10-Aug-06 & Large & Regular & WE,TOT & \\
\hline 3 & Kenny (WE dropped) & 11-Aug-06 & 17-Aug-06 & Small & Regular & WE,TOT & \\
\hline 4 & The Devil Wears Prada & 22-Sep-06 & 28-Sep-06 & Large & Regular & WE,TOT & \\
\hline 5 & World Trade Centre & 29-Sep-06 & 05-Oct-06 & Large & Regular & WE,TOT & \\
\hline 6 & Irresistible & 06-Oct-06 & $12-0 c t-06$ & Small & Art-house & WE,TOT & \\
\hline 7 & Boy Town & 13-Oct-06 & 19-Oct-06 & Small & Regular & WE,TOT & \\
\hline 8 & Suburban Mayhem & 20-Oct-06 & 26-Oct-06 & Small & Art-house & WE,TOT & \\
\hline 9 & God on My Side (Tot Dropped) & 27-Oct-06 & 02-Nov-06 & Small & Art-house & WE,TOT & \\
\hline 10 & Like Minds (Dropped) & 03-Nov-06 & 09-Nov-06 & Small & Art-house & WE,TOT & \\
\hline 11 & The Prestige & 10-Nov-06 & 16-Nov-06 & Large & Regular & WE,TOT & \\
\hline 12 & Hunt Angels (Dropped) & 17-Nov-06 & 30-Nov-06 & Small & Art-house & WE,TOT & \\
\hline 13 & A Scanner Darkly & 24-Nov-06 & 30-Nov-06 & Small & Art-house & WE,TOT & \\
\hline 14 & Charlotte's Web & 01-Dec-06 & 07-Dec-06 & Small & Regular & WE,TOT & \\
\hline 15 & Eragon & 08-Dec-06 & 14-Dec-06 & Large & Art-house & WE,TOT & \\
\hline 16 & Happy Feet & 15-Dec-06 & 26-Dec-06 & Large & Blockbuster & OD,TOT & \\
\hline 17 & Unfinished Sky & 14-Jun-08 & 19-Jun-08 & Small & Art-house & WE,TOT & \\
\hline 18 & Quantum of Solace & 08-Nov-08 & 19-Nov-08 & Large & Blockbuster & WE,TOT & \\
\hline 19 & Australia & 08-Nov-08 & 26-Nov-08 & Large & Blockbuster & WE,TOT & \\
\hline 20 & Centurion & 11-Jul-10 & 29-Jul-10 & Small & Art-house & WE & WE \\
\hline 21 & The Expendables & 11-Jul-10 & 12-Aug-10 & Large & Regular & WE(x2) & WE(x2) \\
\hline 22 & $\begin{array}{l}\text { The Chronicles of Narnia: } \\
\text { The Voyage of the Dawn Treader }\end{array}$ & $11-J u l-10$ & 02-Dec-10 & Large & Blockbuster & WE & WE \\
\hline 23 & The Killer Inside Me & 25-Jul-10 & 26-Aug-10 & Small & Art-house & WE(x2) & WE $(x 2)$ \\
\hline 24 & True Grit & 25-Jul-10 & 26-Jan-11 & Small & Art-house & WE & WE \\
\hline 25 & The Sorcerer's Apprentice & 07-Aug-10 & 09-Sep-10 & Large & Regular & WE $(x 2)$ & $\mathrm{WE}(\mathrm{x} 2)$ \\
\hline 26 & Hall Pass & 07-Aug-10 & 28-Feb-11 & Large & Regular & WE & WE \\
\hline 27 & The Girl Who Played with Fire & 22-Aug-10 & 23-Sep-10 & Small & Art-house & WE $(x 2)$ & WE $(x 2)$ \\
\hline 28 & Mars Needs Moms & 22-Aug-10 & 14-Apr-11 & Large & Blockbuster & WE & WE \\
\hline 29 & Eat Pray Love & 05-Sep-10 & 07-Oct-10 & Large & Regular & WE $(x 2)$ & $\mathrm{WE}(\mathrm{x} 2)$ \\
\hline 30 & Scream 4 & 05-Sep-10 & 14-Apr-11 & Large & Regular & WE & WE \\
\hline 31 & Paranormal Activity 2 & 20-Sep-10 & 21-Oct-10 & Large & Regular & WE $(x 2)$ & WE $(x 2)$ \\
\hline 32 & $\begin{array}{l}\text { Pirates of the Caribbean: } \\
\text { On Stranger Tides }\end{array}$ & 20-Sep-10 & 19-May-11 & Large & Blockbuster & WE & WE \\
\hline 33 & Oceans (Dropped) & 03-Oct-10 & 19-May-11 & Small & Art-house & WE $(x 2)$ & $W E(x 2)$ \\
\hline 34 & X-Men: First Class & 03-Oct-10 & 02-Jun-11 & Large & Blockbuster & WE & WE \\
\hline 35 & $\begin{array}{l}\text { Harry Potter and the Deathly Hallows: } \\
\text { Part } 1\end{array}$ & $17-O c t-10$ & 18-Nov-10 & Large & Blockbuster & WE & \\
\hline 36 & Horrible Bosses & 17-Oct-10 & 25-Aug-11 & Small & Regular & WE & \\
\hline 37 & Romantics Anonymous & 13-Apr-12 & 19-Apr-12 & Small-Med & Art-house & WE & \\
\hline 38 & The Lucky One & 13-Apr-12 & 19-Apr-12 & Small-Med & Regular & WE & \\
\hline 39 & The Avengers & 20-Apr-12 & 25-Apr-12 & Med-Large & Blockbuster & WE & \\
\hline 40 & Irvine Welsh's Ecstasy & 20-Apr-12 & 26-Apr-12 & Small-Med & Art-house & WE & \\
\hline 41 & W.E. & 27-Apr-12 & 03-May-12 & Small-Med & Art-house & WE & \\
\hline 42 & Delicacy & 27-Apr-12 & 03-May-12 & Small-Med & Art-house & WE & \\
\hline 43 & Dark Shadows & 04-May-12 & 10-May-12 & Med-Large & Blockbuster & WE & \\
\hline 44 & What to Expect When You're Expecting & 04-May-12 & 31-May-12 & Med-Large & Regular & WE & \\
\hline 45 & The Five-Year Engagement (Dropped) & 11-May-12 & 03-May-12 & Med-Large & Regular & WE & \\
\hline 46 & Safe(Dropped) & 11-May-12 & 03-May-12 & Med-Large & Regular & WE & \\
\hline 47 & Bel Ami & 18-May-12 & 24-May-12 & Small-Med & Art-house & WE & \\
\hline 48 & Men in Black 3 & 18-May-12 & 24-May-12 & Large & Blockbuster & WE & \\
\hline 49 & Declaration of War & 25-May-12 & 31-May-12 & Small & Art-house & WE & \\
\hline
\end{tabular}




\section{Appendix A: Table of All Films (Cont)}

\begin{tabular}{|c|c|c|c|c|c|c|c|}
\hline ID & Title & $\begin{array}{l}\text { Experiment } \\
\text { Date }\end{array}$ & $\begin{array}{l}\text { Release } \\
\text { Date }\end{array}$ & Bucket Size & $\begin{array}{l}\text { Analysis } \\
\text { Definition }\end{array}$ & $\begin{array}{l}\text { BOP } \\
\text { (OD, WE, TOT) }\end{array}$ & $\begin{array}{l}\text { GOG } \\
\text { (WE, TOT) }\end{array}$ \\
\hline 50 & Get the Gringo & 25-May-12 & 31-May-12 & Med-Large & Regular & WE & \\
\hline 51 & Prometheus & 01-Jun-12 & 07-Jun-12 & Large & Blockbuster & WE & \\
\hline 52 & Friends with Kids & 01-Jun-12 & 07-Jun-12 & Small-Med & Art-house & WE & \\
\hline 53 & Rock of Ages & 08-Jun-12 & 14-Jun-12 & Large & Regular & WE & \\
\hline 54 & That's My Boy & 08-Jun-12 & 14-Jun-12 & Small-Med & Regular & WE & \\
\hline 55 & A Royal Affair & 15-Jun-12 & 21-Jun-12 & Small & Art-house & WE & \\
\hline 56 & Snow White and the Huntsman & 15-Jun-12 & 21-Jun-12 & Large & Blockbuster & WE & \\
\hline 57 & Brave & 15-Jun-12 & 21-Jun-12 & Large & Blockbuster & WE & \\
\hline 58 & The Dark Knight Rises & 13-Apr-12 & 19-Jul-12 & Extra Large & Blockbuster & WE & TOT \\
\hline 59 & Gl Joe: Retaliation & 13-Apr-12 & 28-Mar-13 & NA & & & TOT \\
\hline 60 & Premium Rush & 20-Apr-12 & 08-Nov-12 & NA & & & TOT \\
\hline 61 & Hotel Transylvania & 20-Apr-12 & 20-Sep-12 & NA & & & TOT \\
\hline 62 & Savages & 27-Apr-12 & $18-$-Oct-12 & NA & & & TOT \\
\hline 63 & Taken 2 & 27-Apr-12 & $04-0 c t-12$ & NA & & & TOT \\
\hline 64 & Lawless & 04-May-12 & 11-Oct-12 & NA & & & TOT \\
\hline 65 & The Watch & 04-May-12 & 13-Sep-12 & NA & & & TOT \\
\hline 66 & Argo & 11-May-12 & $25-$ Oct-12 & NA & & & TOT \\
\hline 67 & Gangster Squad & 11-May-12 & 10-Jan-13 & NA & & & TOT \\
\hline 68 & Skyfall & 18-May-12 & 22-Nov-12 & NA & & & TOT \\
\hline 69 & $\begin{array}{l}\text { The Twilight Saga: } \\
\text { Breaking Dawn Part } 2\end{array}$ & 18-May-12 & 15-Nov-12 & NA & & & TOT \\
\hline 70 & Gravity & 25-May-12 & 03-Oct-13 & NA & & & TOT \\
\hline 71 & 47 Ronin & 25-May-12 & 16-Jan-14 & NA & & & TOT \\
\hline 72 & Rise of the Guardians & 01-Jun-12 & 13-Dec-12 & NA & & & TOT \\
\hline 73 & Here Comes the Boom & 01-Jun-12 & 06-Dec-12 & NA & & & TOT \\
\hline 74 & Ted & 08-Jun-12 & 05-Jul-12 & NA & & & TOT \\
\hline 75 & The Hobbit: An Unexpected Journey & 08-Jun-12 & 26-Dec-12 & NA & & & TOT \\
\hline 76 & Life of Pi & 15-Jun-12 & 01-Jan-12 & NA & & & TOT \\
\hline 77 & Les Miserables & 15-Jun-12 & 26-Dec-12 & NA & & & TOT \\
\hline
\end{tabular}

Notes: "OD" is opening day box office, "WE" is opening weekend box office, and "TOT" is total box office. 


\section{Appendix B: Instructions}

\section{Welcome Everyone}

Box Office Prophecy is about to start - and to ensure everyone has the information they need to be big winners I have put together this little welcome pack for you all.

Box Office Prophecy is a fun, interactive process testing your ability to predict box office revenues for selected films playing in Australian cinemas.

This year we are doing something a little different, so even if you have participated in BOP before you may want to read through the HOW TO PARTICIPATE section to familiarize yourself with the process and increase your chances of making money.

Box Office Prophecy is ultimately a research project for Caltech and Sydney University in association with AFTRS to assess the nature of information that exists regarding the potential success or failure of theatrically released films.

However it's also loads of fun and FINANCIALLY rewarding. We have $\$ 2,000$ in prize money every week. So gather your information, look over the decisions of others and make as much money as you can.

We have a great range of films this year - everything from small French art house releases to huge US blockbusters - so you'll have the chance to test yourselves on your knowledge of all different genres, subject matters and styles of films.

There is one big omission! You may notice in the list of films there is a distinct lack of Australian productions. This is regrettable and everything was done to try and include some local Australian productions onto the list but unfortunately we simply could not get the information we needed regarding screen numbers and distribution dates for the Australian films that did fit within the BOP schedule.

That said several talented Aussies have contributed to the films on the list including directors Scott Hicks and Christopher Nolan as well as actors Abbie Cornish, Russell Crowe, Hugh Jackman and Chris Hemsworth. Also, one of the week nine films, Ted, was partly funded and made in Australia.

The rest of this pack gives you further information regarding how to participate and specific information regarding the cast, crew and advanced 'buzz' of the selected films. Don't be afraid to do your own research though - the people who won big last BOP made sure they were well informed.

Good luck and- 'May the BOP be with you.'

Gabiann Marin

The Big BOPper 


\section{When do we start?}

The date has been chosen. The red carpet has been laid and the Box Office Prophecy is just around the corner.

\section{BOP goes live on Friday the $13^{\text {th }}$ of April 2012 (what an auspicious day!)}

On that day - and every following Friday - you will have one hour to make your choices regarding the box office revenue outcomes of the selected films.

BOP opens at $12 \mathrm{pm}$ sharp and closes at $1 \mathrm{pm}$.

\section{How to Participate}

\section{Short Range vs. Long Range films}

You will notice that each week there are two films which are opening the following weekend, and two films which are releasing many months away.

These are referred to as short range films (released the following week) and long range films (released 3 -6 months away) and you place your selections quite differently for each.

When you login you will initially be directed to a screen which asks you about long range films.

\section{Long Range Films}

The long range predictions are for films which are to be released anywhere from 3-6 months from the BOP date. Predictions for these films work differently from short range films. Firstly, they involve making a prediction about 'total' lifetime box office (up to four months from initial release). Secondly, rather than participating in buying tickets like the short-range films discussed below, you are asked the following question:

"In the blank beside each long range film, please provide a guess about the total lifetime Australian box office (up to four months). For each film, the person whose guess is closest to the median (half of the guesses are above and half are below) of all the guesses for that film will win \$300."

Notice that you are not being paid on what the box office turns out to be. You are being paid if you give us an accurate prediction of what people think that the box office will be. 


\section{Short Range Films}

Once you have completed the long range film questions, you enter the BOP prediction environment for short range films where your objective is to predict 'opening weekend' (Thursday - Sunday) box office revenues. Each week there will be two films in the short range selection.

Every registered player will be given 500 BOP dollars for each short range film each week.

You will see that you can buy tickets for specific box office ranges, which we call 'buckets'.

The buckets have been determined based on the number of opening screens for the film. And each film has been categorized as one of the following:

- Art house release: less than 50 opening screens

- Small release : $50-100$ opening screens

- Regular release: 100 - 250 opening screens

- Blockbuster release: more than 250 opening screens

You buy tickets in as many buckets as you like. In buying tickets you should consider what you think the chances are that a bucket will win and the number of tickets that others have bought on the bucket; because you are sharing the winnings in proportion to holdings. You can spread your investment across a number of potential outcome buckets and remember that the least likely might return the largest return if you are the only one investing in that bucket.

As the hour progresses you will be able to see where others are buying tickets and will be able to see if there is any specific trend happening. However be careful, the longer you wait the more expensive the tickets become, i.e. at the beginning of the hour the ticket prices are low but after a short period the price per ticket will increase. So, as more tickets are purchased and trends become evident, the cost of a ticket will increase such that near the end of the hour your 500 BOP dollars will not buy very many tickets. Think strategically, go with your gut or just take a punt; the choice is yours but whatever you decide make sure you make all purchases within that hour window (12pm - 1pm Friday). A ticket, once purchased, cannot be returned, so think carefully about where you place your money.

You should note that BOP money has no outside value, so you should spend it all.

The BOP interface provides information on the following (see next page for further detail):

- The time remaining in the experiment

- The current price of a ticket

- The total number of tickets sold

- Your remaining budget

- The number of tickets you hold in each bucket

- The total number of tickets held by all participants in each bucket

- The percent of tickets you own within a particular bucket

- The percent of total tickets sold in a particular bucket of all tickets sold 
As well there are useful graphics included to assist you in interpreting where others are purchasing tickets relative to your own purchases. Specifically, there are orange (horizontal) bars which correspond to the percent of total tickets sold in a particular bucket of all tickets sold. Also, there are green (horizontal) bars which represent the percentage of each bucket's tickets which you own.

Prizes are awarded corresponding to the proportion of tickets you hold in the winning bucket. For example, if (as in the screen shot below) the winning bucket is $\$ 90,000-\$ 119,999$, you own $60 \%$ of all tickets purchased (i.e. 3 out of the 5 tickets) and are paid $60 \%$ of the total prize pool.

The picture below shows the key features of the BOP interface screen:
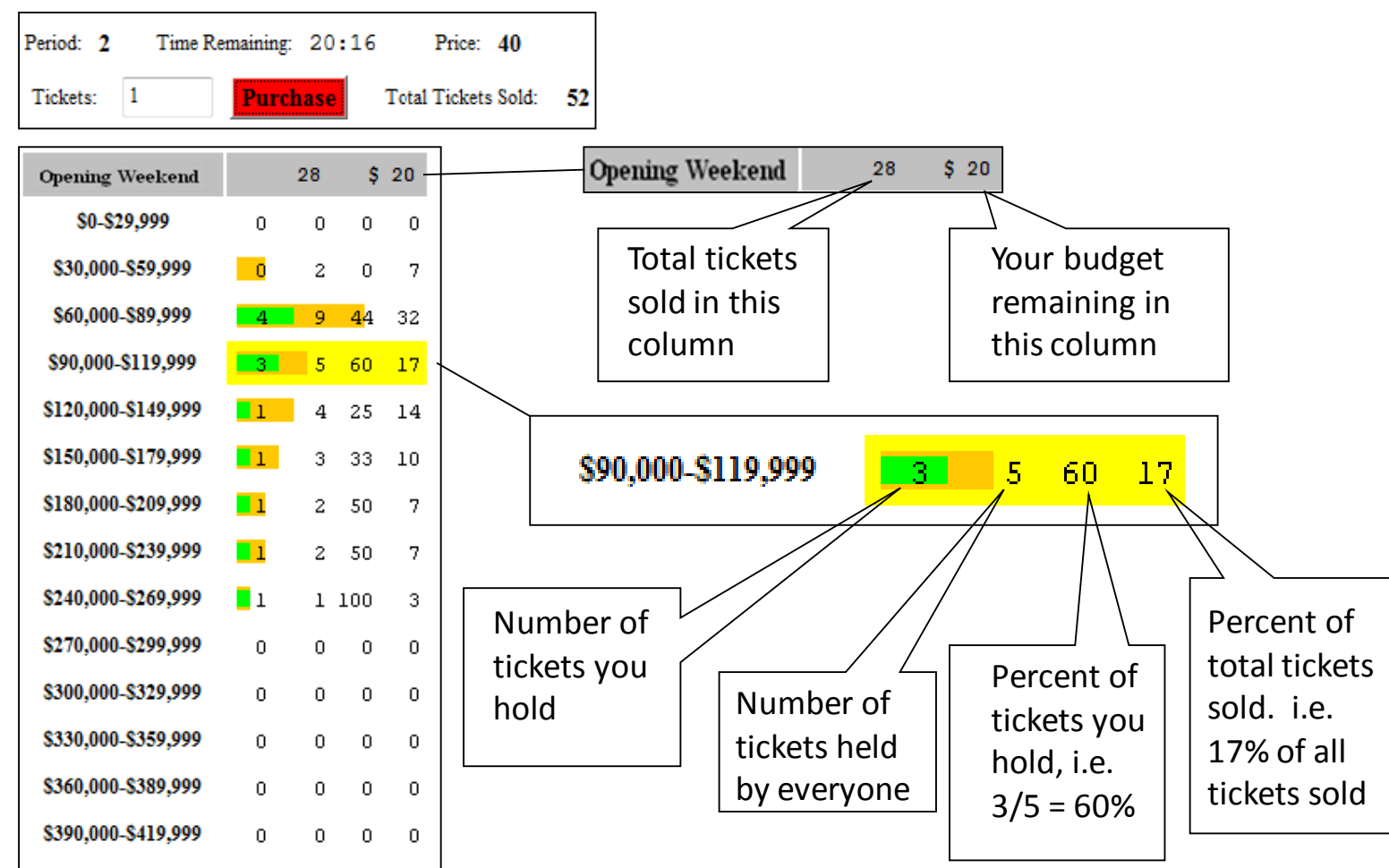

$\$ 420,000-\$ 449,999 \quad 0 \quad 00000$

$\$ 450,000+\quad 0 \quad 00000$

The orange distribution is the implied probability of all ticket purchases and the green is your choices. Notice that these are represented like a (horizontal) bar graph. 


\section{The serious stuff}

$\mathrm{BOP}$ is a serious business, and there is serious money to be won so we have to make sure that the rules of the game are understood and followed.

The rules:

1. You must be a registered player to participate.

2. BOP is only open the Fridays from $12 \mathrm{pm}-1 \mathrm{pm}$. BOP will close precisely at $1 \mathrm{pm}$.

3. Each participant makes one guess for each long range film's total box office for that week's session. Once the amount is entered it cannot be changed. The winner of the prize money will be the person whose guess is the closest to the median of all the long range guesses. In the event that there is a tie, the prize money will be shared equally.

4. Each week you will be given another 1,000 BOP dollars, i.e. 500 for each short range film. There is no roll over from unused dollars in previous sessions. Any unused (not used to buy tickets) BOP Dollars will be erased from your account at the end of each BOP session.

5. Actual Box Office returns will be calculated based on opening weekend revenues (ThursdaySunday takings) as advised by the MPDAA- advanced screenings, special screenings and event premieres will not be counted in the box office return. A film has to be released in all major states (NSW, VIC, QLD) to be considered as officially released.

6. The Monday after the opening weekend of each short range film we will announce the actual amount of the box office and anyone who placed bets in the winning range will share in the prize money for that week based on the proportion of their ticket holdings.

7. Winners will not be announced by name; although we will alert all registered players of how many winners there were for each session as well as the actual box office of the selected films.

8. Winners will be alerted privately and winnings will be directly deposited into the bank account provided at registration. If you need to change or update your bank account please do so as soon as possible (see FAQ)

9. Prize money is $\$ 2,000$ per week distributed amongst the winners in the following way: $\$ 700$ per film for each short range film distributed to winner(s) in relation to the value of their winning bets. $\$ 300$ per film for each long range winner(s). It is assumed there will usually only be one long range winner per week although should there be multiple winners the $\$ 300$ will be shared equally between them.

Further Terms and conditions regarding BOP can be found on the website http://eeps6.caltech.edu/boxoffice, by clicking 'Technical FAQ'. 


\section{Practices, Logging on and registering}

Go to the home site http://eeps6.caltech.edu/boxoffice, and click on 'Want to practice?'

Here you will be able to log onto the site and practice placing bets and see how the interface works. On this site, you can also access information about the list of films and the film schedule.

We strongly recommend you PRACTICE before the market officially opens on the 13/4/2012.

\section{FAQ}

\section{Can I practice before the official site opens?}

Yes, the practice market is open now. Go to http://eeps6.caltech.edu/boxoffice, and click on 'Want to practice?'

\section{How will I know if I have won?}

You will be alerted through the email address you have provided.

\section{What if I can't sign in?}

If you have trouble logging in you can click on 'I forgot my password 'under the sign in field on the main sign in page. Once prompted put in your email address and last name and you will be able to reset your password and enter the site.

If this still doesn't work you should contact Gabiann Marin at gabiann.marin@aftrs.edu.au as soon as possible

It is a good idea to check that you can get into the site a day or two before the BOP sessions as you only have 1 hour to sign in and make your choices and you may not have your matter resolved in time if you leave it to the day or the hour of the session.

\section{Can I save BOP Dollars to use for later sessions?}

No. You must use the BOP dollars in each session. Any BOP dollars not used will be erased. Everyone will start with exactly the same number of BOP Dollars each session regardless of how many the spent or if they won real money, in previous sessions.

\section{What if I miss a session?}

You do not have to play every session. If you miss a session you can simply log in the next session and play on. You cannot 'make up' a session. Once a BOP session is closed it is impossible for any choices to be entered for those films including long range guesses. 


\section{How can I increase my chances of winning?}

Play every session and try to get as much information about the films as possible. You aren't trying to work out the biggest grossing films, just what you think individual films may make at the opening box office.

Some handy hints include

1. Look up similar films opening box office returns. Similar may mean alike in style, content, screening numbers or have similar or the same creative or performance people.

2. Check out the links provided, and don't be afraid of doing your own research - you may encounter something that will make a huge difference to your guesses.

\section{Who can I contact if any of my contact or bank details change?}

Contact Gabiann Marin at AFTRS as soon as possible to ensure you can receive information and winnings. Her direct email is gabiann.marin@aftrs.edu.au.

If you have any other questions or concerns please email me directly at gabiann.marin@aftrs.edu.au and I'll try to resolve it as soon as possible. Please be advised I am not in every day so problems may take a few days to resolve- however I will try to get things sorted as soon as possible for you. 


\section{ADDITIONAL MATERIALS: GENERAL INSTRUCTIONS}

Key variables

Dear Team,

Please find attached the final list of films for BOP. We will send an updated file with film links, etc. in due course.

Please note we have our first session Friday April 13, 12-1pm (AEST). In total there will be 10 weekly sessions, each of which will run Fridays, 12-1pm.

As you will see from the attached spreadsheet, each week we have four films. Two of these are short range (opening the following week) and the other two are long range (opening 3-6 months).

For the short range films, we use the 'pari-mutuel' prediction mechanism for opening weekend revenues (Thursday - Sunday).

For the long range films, we use the 'average guess' prediction mechanism for cumulative revenues (up to four months from release).

For each film we have listed 'release type' as one of the following:

1. Art house ( $<50$ opening screens)

2. Small (50-100 opening screens)

3. Regular (100-250 opening screens)

4. Blockbuster (>250 opening screens)

The 'release type' is particularly important for the short-range films as it dictates the range of buckets provided on the pari-mutuel screen. Please refer to the 'BUCKETS' tab on the attached spreadsheet to see the corresponding divisions.

Over the 10 week course of the experiment, we will be giving away $A \$ 20,000$. Therefore, each week we give away $\mathbf{A} \mathbf{\$ 2}, \mathbf{0 0 0}$. For each short-range film, the pari-mutuel prize pool is $\mathbf{A} \mathbf{\$ 7 0 0}$. For each long-range film, the prize is $\mathbf{A} \mathbf{\$ 3 0 0}$. Note that we would generally expect only one participant to claim the long range prize, but a number of participants may share in the short-range prize pool.

Please feel free to contact me should you have any questions. 


\section{ADDITIONAL MATERIALS: FILMS}

\begin{tabular}{|c|c|c|c|c|c|c|c|}
\hline \multirow[b]{2}{*}{ Round } & \multirow[b]{2}{*}{ BOP date } & \multicolumn{6}{|c|}{ Short range films } \\
\hline & & Film 1 & Film 1 date & Film 1 type & Film 2 & Film 2 date & Film 2 type \\
\hline 1 & $13-04-12$ & Romantics Anonymous & $19-04-12$ & art house & The Lucky One & $19-04-12$ & regular \\
\hline 2 & $20-04-12$ & The Avengers & $25-04-12$ & blockbuster & Irvine Welsh's Ecstasy & $26-04-12$ & art house \\
\hline 3 & 27-04-12 & W.E & $03-05-12$ & art house & Delicacy & $03-05-12$ & art house \\
\hline 4 & 04-05-12 & Dark Shadows & $10-05-12$ & blockbuster & What to Expect When You're Expecting & $10-05-12$ & regular \\
\hline 5 & $11-05-12$ & Five-Year Engagement, The & $17-05-12$ & regular & Safe & $17-05-12$ & regular \\
\hline 6 & $18-05-12$ & Bel Ami & 24-05-12 & art house & Men in Black 3 & 24-05-12 & blockbuster \\
\hline 7 & $25-05-12$ & Think Like A Man & $31-05-12$ & regular & Get the Gringo & $31-05-12$ & small \\
\hline 8 & 01-06-12 & Prometheus & $07-06-12$ & blockbuster & Friends with Kids & $07-06-12$ & art house \\
\hline 9 & 08-06-12 & Rock of Ages & $14-06-12$ & blockbuster & That's My Boy & $14-06-12$ & regular \\
\hline \multirow[t]{2}{*}{10} & $15-06-12$ & A Royal Affair & 21-06-12 & art house & Snow White and the Huntsman & $21-06-12$ & blockbuster \\
\hline & & \multicolumn{6}{|c|}{ Long range films } \\
\hline Round & BOP date & Film 3 & Film 3 date & Film 3 type & Film 4 & Film 4 date & Film 4 type \\
\hline 1 & $13-04-12$ & Dark Knight Rises, The & $19-07-12$ & blockbuster & GI Joe: Retaliation & $19-07-12$ & regular \\
\hline 2 & 20-04-12 & Premium Rush & $13-09-12$ & regular & Hotel Transylvania & 20-09-12 & blockbuster \\
\hline 3 & 27-04-12 & Savages & 27-09-12 & small & Taken 2 & $04-10-12$ & regular \\
\hline 4 & 04-05-12 & Wettest County & 06-09-12 & regular & Neighbourhood Watch & $13-09-12$ & blockbuster \\
\hline 5 & $11-05-12$ & Argo & $27-09-12$ & regular & Gangster Squad & $01-11-12$ & regular \\
\hline 6 & $18-05-12$ & Bond 23 & $22-11-12$ & blockbuster & The Twilight Saga: Breaking Dawn - Part 2 & $15-11-12$ & blockbuster \\
\hline 7 & $25-05-12$ & Gravity & 29-11-12 & regular & 47 Ronin & $29-11-12$ & blockbuster \\
\hline 8 & 01-06-12 & Rise of the Guardians & $13-12-12$ & blockbuster & Here Comes The Boom & $06-12-12$ & blockbuster \\
\hline 9 & 08-06-12 & Ted & $07-12-12$ & regular & Hobbit: An Unexpected Journey, the & $26-12-12$ & blockbuster \\
\hline 10 & $15-06-12$ & Life Of Pi & $20-12-12$ & blockbuster & Les Miserables & $26-12-12$ & regular \\
\hline
\end{tabular}

Notes:

1) Short range film predictions are for 'opening weekend' revenues (defined Thursday - Sunday inclusive).

2) Long range film predictions are for 'cumulative' (i.e. life-time) revenue up to 4 months. 